\title{
Priming is key to effective incorporation of image-guided thermal ablation into immunotherapy protocols
}

Matthew T. Silvestrini, ${ }^{1}$ Elizabeth S. Ingham, ${ }^{1}$ Lisa M. Mahakian, ${ }^{1}$ Azadeh Kheirolomoom, ${ }^{1}$ Yu Liu, ${ }^{1}$ Brett Z. Fite, ${ }^{1}$ Sarah M. Tam,, ${ }^{1}$ Samantha T. Tucci,, Katherine D. Watson, ${ }^{1}$ Andrew W. Wong, Arta M. Monjazeb, ${ }^{2}$ Neil E. Hubbard, ${ }^{3}$ William J. Murphy, ${ }^{4}$ Alexander D. Borowsky, ${ }^{3}$ and Katherine W. Ferrara ${ }^{1}$

Department of Biomedical Engineering, 2Department of Radiation Oncology, ${ }^{3}$ Center for Comparative Medicine, ${ }^{4}$ Department of Dermatology, Institute for Regenerative Cures, University of California, Davis, California, USA.

Focal therapies play an important role in the treatment of cancers where palliation is desired, local control is needed, or surgical resection is not feasible. Pairing immunotherapy with such focal treatments is particularly attractive; however, there is emerging evidence that focal therapy can have a positive or negative impact on the efficacy of immunotherapy. Thermal ablation is an appealing modality to pair with such protocols, as tumors can be rapidly debulked (cell death occurring within minutes to hours), tumor antigens can be released locally, and treatment can be conducted and repeated without the concerns of radiation-based therapies. In a syngeneic model of epithelial cancer, we found that 7 days of immunotherapy (TLR9 agonist and checkpoint blockade), prior to thermal ablation, reduced macrophages and myeloid-derived suppressor cells and enhanced IFN- $\gamma$-producing CD8 ${ }^{+} \mathrm{T}$ cells, the M1 macrophage fraction, and PD-L1 expression on $\mathrm{CD}^{+} 5^{+}$cells. Continued treatment with immunotherapy alone or with immunotherapy combined with ablation (primed ablation) then resulted in a complete response in $80 \%$ of treated mice at day 90, and primed ablation expanded CD8 ${ }^{+} \mathrm{T}$ cells as compared with all control groups. When the tumor burden was increased by implantation of 3 orthotopic tumors, successive primed ablation of 2 discrete lesions resulted in survival of $60 \%$ of treated mice as compared with $25 \%$ of mice treated with immunotherapy alone. Alternatively, when immunotherapy was begun immediately after thermal ablation, the abscopal effect was diminished and none of the mice within the cohort exhibited a complete response. In summary, we found that immunotherapy begun before ablation can be curative and can enhance efficacy in the presence of a high tumor burden. Two mechanisms have potential to impact the efficacy of immunotherapy when begun immediately after thermal ablation: mechanical changes in the tumor microenvironment and inflammatory-mediated changes in immune phenotype.

Conflict of interest: The authors have declared that no conflict of interest exists.

Submitted: September 6, 2016 Accepted: February 3, 2017 Published: March 23, 2017

Reference information: JCI Insight. 2017;2(6):e90521. https:// doi.org/10.1172/jci.insight.90521.

\section{Introduction}

Local ablative therapies such as high-intensity focused ultrasound, ablative radiotherapy, radiofrequency ablation, and cryotherapy are increasing in clinical use. As patients are living longer with metastatic disease, such therapies provide local control and palliation of tumors. In recent years, the extension of survival in patients with metastatic disease is due in part to the success of new immunotherapy strategies, including checkpoint inhibition $(1,2)$. The administration of a checkpoint blockade has yet to be refined; many questions remain about the optimal scheduling and dosing for specific cancers and the biomarkers that are associated with disease outcome (3). Multiple strategies to enhance the response of checkpoint inhibitors have been proposed, including additional systemic chemotherapies (4-6) or immunotherapies $(7,8)$ and local therapies $(9,10)$. There is a growing interest in combining immunotherapy with ablative strategies, but little data is available to guide if and how these modalities should be combined (9). Our goal is to combine imaging, immunological, and histopathological assays to determine whether mechanical and immunologic changes in the tumor microenvironment resulting from focal ablation impact the window of opportunity for employing immunotherapy. 
Local radiotherapy, thermal therapy and TLR agonists can elicit immunogenic cell death (ICD) to potentiate the clonal expansion of tumor-specific T cells (9). Each stimulates some degree of immunization to tumor-associated antigens following treatment $(11,12)$; however, each has proven insufficient to overcome tumor-mediated immunosuppression, thus limiting the abscopal effect and the ability to treat metastatic disease $(9,13-16)$. We specifically explore the implementation of magnetic resonance-guided focused ultrasound (MRgFUS) ablation combined with TLR agonists and checkpoint inhibitors. The clinical indications for MRgFUS have exploded in recent years due to the opportunity to precisely deliver treatment to select regions of interest (17-19); thermal ablation of large regions is clinically targeted by scanning the ultrasound focus in a circular pattern or creating a grid pattern of overlapping ablation points $(20,21)$. Treatment is monitored by MRI and thermometry, which are used to direct the ultrasound beam and generate maps of the temperature and thermal dose in real-time (22-24). Here, the ultrasound beam was electronically focused in the tumor to a volume of $0.5 \times 0.5 \times 1.5 \mathrm{~mm}^{3}$ and rapidly swept through the tumor volume to accomplish a treatment plan developed by the operator.

Thermal ablative therapies have the potential to speed cell death as compared with radiotherapy. While the full measure of ICD resulting from radiotherapy requires weeks to evaluate completely, the effects of heat-mediated ablation are nearly immediate (25). Response to current immunotherapy protocols also requires months to be realized; thermal ablation has the potential to offer rapid response and palliation. Such treatments can be repeated on a schedule that can be optimized for each patient without concern for radiation-mediated toxicities. Here, we create thermoablative-immunotherapy (TA-immunotherapy) protocols by combining anti-PD-1 ( $\alpha$ PD-1) therapy with a TLR agonist $(\mathrm{CpG})$ and ablation, and we compare protocols in which immunotherapy begins before or after ablation. We evaluate the combined protocols in the neu exon deletion line (NDL) syngeneic, orthotopic breast cancer model of epithelial, focal, mammary adenocarcinoma $(26,27)$. Given the growing use of immunotherapy and focal therapies in the clinic and in combining these modalities, these studies have profound and direct clinical implications for how immunotherapy should be combined with ablative strategies.

\section{Results}

MRgFUS ablation induces rapid, ICD to transiently suppress local tumor growth. Prior to treatment, we first quantified the native tumor infiltrating lymphocyte population by flow cytometry at day 28 after implantation ( $\sim 5 \mathrm{~mm}$ in diameter). Leukocytes (CD45 cells) represented $3.6 \% \pm 2.2 \%$ of the live cells and were comprised of the following as a fraction of $\mathrm{CD} 45^{+}$cells: $\mathrm{CD} 4^{+} \mathrm{T}$ cells $(7.3 \% \pm 3.8 \%), \mathrm{CD} 8^{+} \mathrm{T}$ cells $(20.7 \% \pm$ $10.3 \%)$, IFN- $\gamma$-producing CD4 $4^{+} \mathrm{T}$ cells $(0.77 \% \pm 0.34 \%)$, IFN- $\gamma$-producing CD8 ${ }^{+} \mathrm{T}$ cells $(2.3 \% \pm 1.34 \%)$, macrophages $(15.6 \% \pm 2.8 \%)$, myeloid-derived suppressor cells (MDSCs) $(3.3 \pm 2.2 \%)$, DCs $(21.8 \% \pm$ $3.5 \%)$, and NK cells $(15.3 \% \pm 12.4 \%)$.

To characterize the immune effects of MRgFUS ablation on treated and distant tumors, we analyzed tumor growth, changes in immunocyte populations, and tumor histology after a schedule of thermal dosing. Two treatments of MRgFUS ablation (Figure 1, A-C) to a discrete region within a single tumor in bilaterally transplanted mice were performed on days 21 and 28 after tumor transplantation. MRgFUS provided the opportunity to tailor the treatment to the tumor region of interest by mechanically scanning the ultrasound focus in a circle that conformed to the tumor diameter and monitoring both the local temperature increase and overall thermal dose. Thermal ablation was sufficient to suppress the treated tumor growth (Figure 1D) compared with the no-treatment control (Figure 1F); 43\% (3 of 7) of the treated tumors exhibited a reduced growth rate. This effect was transient, however, and by day 40, all treated tumors began to progress. Ultimately, MRgFUS ablation did not significantly alter the average contralateral tumor growth (Figure 1E) compared with the no-treatment control and therefore did not impact mean survival.

To elucidate the immune effects responsible for local tumor suppression after thermal dosing, we performed flow cytometry at day 28 , prior to the second application of thermal ablation. Significant increases in IFN- $\gamma$-secreting CD4 $4^{+} \mathrm{T}$ cells (IFN- $\gamma \mathrm{CD} 4^{+} \mathrm{T}$ cells) (Figure $1 \mathrm{G}$ ) and cytotoxic CD8 ${ }^{+} \mathrm{T}$ cells $\left(\mathrm{CD} 8^{+} \mathrm{T}\right.$ cells) (Figure 1H), as well as a significant decrease in Tregs (Figure 1I), were observed in the treated tumor only. Further, we evaluated high mobility group box 1 (HMGB1) protein release (a damage-associated molecular pattern capable of activating TLR4 signaling) in NDL cells in vitro to assess changes likely to result in signaling related to ICD. Specifically, we found that thermal dosing was capable of eliciting ICD, as indicated by the release of HMGB1, with an effect that peaked near $60^{\circ} \mathrm{C}$ and was similar to the wellknown effect of the anthracycline doxorubicin (Figure 1J). 
A

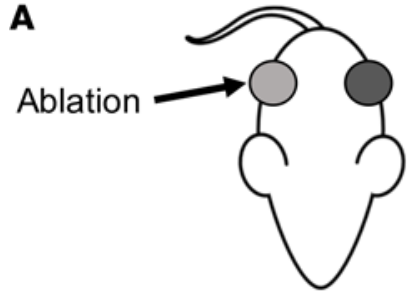

D

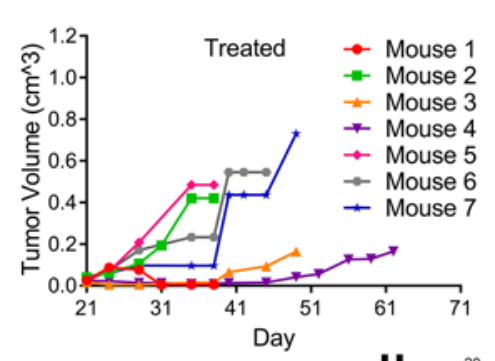

G

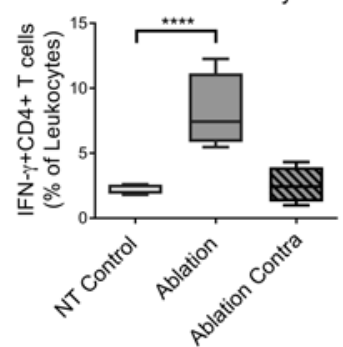

H

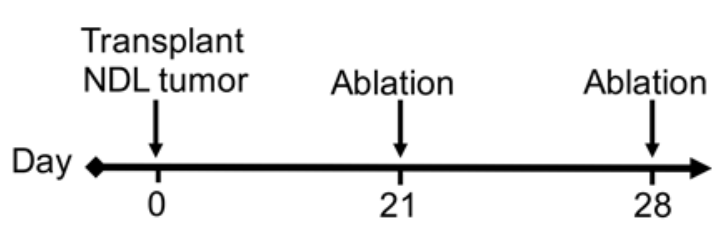

E
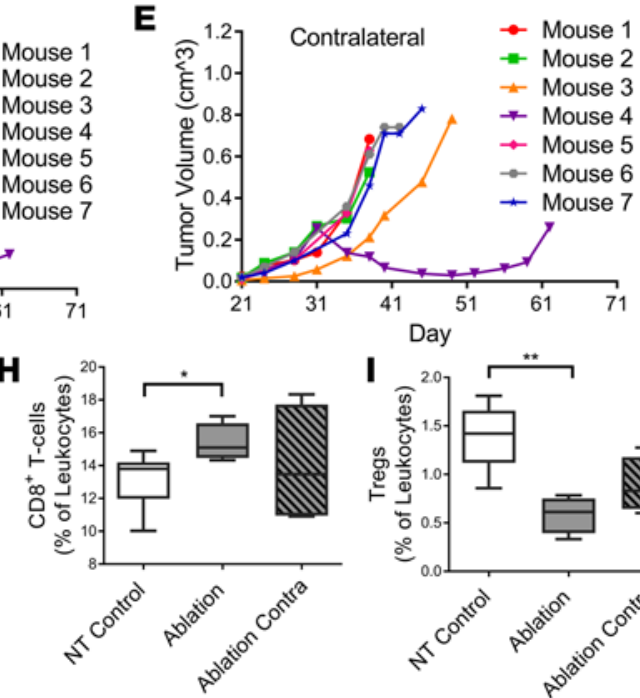

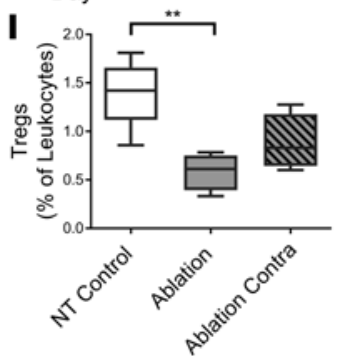

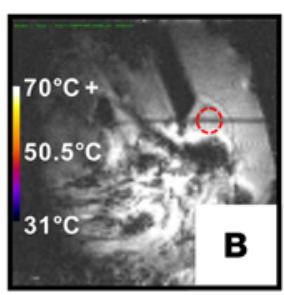

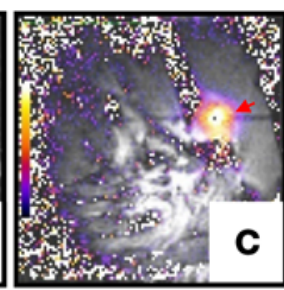

F

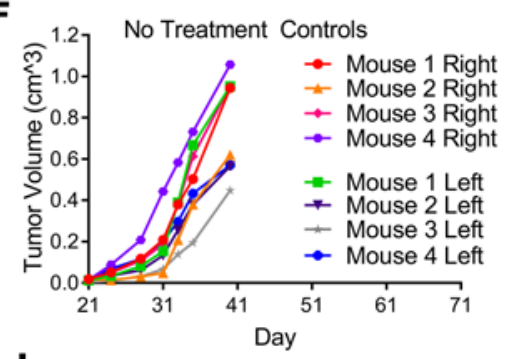

J

HMGB1 Release

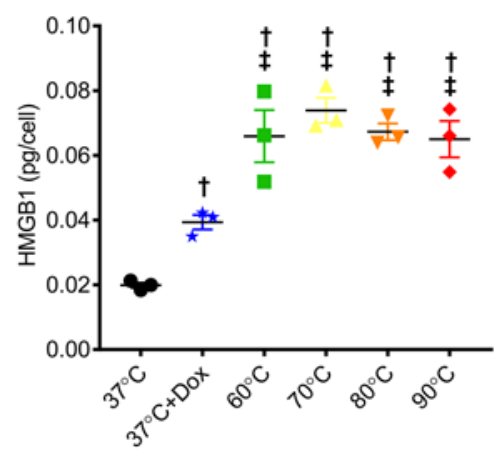

$3^{10}$
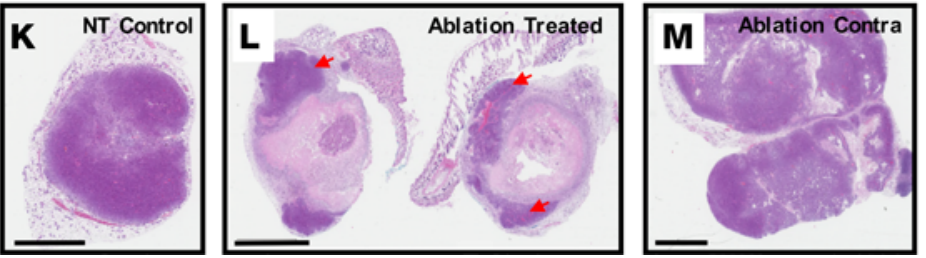

Figure 1. MRgFUS ablation promotes local antitumor immune response. (A) Regimen of thermal ablation in mice orthotopically transplanted with neu deletion line (NDL) tumor biopsies in the fourth and ninth mammary fat pad. (B and C) Magnetic resonance-guided focused ultrasound (MRgFUS) ablation protocol and temperature mapping for thermal ablation in vivo (white color indicates minimum threshold of $70^{\circ} \mathrm{C}$ ). (B) T1w images of localized region to be ablated (red dotted circle). (C) MR thermometry image following ablation protocol (red arrow indicates heated region). (D-F) NDL tumor growth following MRgFUS ablation $(n=7)$ compared with no-treatment (NT) control NDL tumor growth ( $n=34$ total, 8 representative growth curves shown for this individual study). Mice per group examined in 3 separate experiments. (D) Thermally ablated tumors exhibited a transient suppression in growth compared with (E) contralateral and (F) NT control tumors. (G-I) The entire fourth and ninth mammary fat pad (tumor and embedded lymph node) was harvested at day 28 , and immune cells were quantified by flow cytometry ( $n=4$ per group). Effect of therapy on (C) IFN- $\gamma$ CD4 ${ }^{+}$T cells, (H) CD8 ${ }^{+}$T cells, and (I) Tregs in the treated and contralateral tumors. For box-and-whiskers plots, the whiskers represent the minimum and maximum values, the box boundaries represent the 25th and 75 th percentiles, and the middle line is the median value. (J) High mobility group box 1 (HMCB1) protein release in vitro quantified via ELISA. Treatment with doxorubicin (Dox) at $37^{\circ} \mathrm{C}$ represents a positive control. Each dot represents a sample. Data are mean \pm SEM of 3 measurements. (K-M) H\&E staining performed at day 31 confirmed that, compared with (K) control tumors $(n=3)$, viable tumor was reduced in $(\mathbf{L})$ treated tumors $(n=3)$, where residual viable tumor tissue (red arrows) existed outside the path of the ultrasound beam. Thermal ablation did not reduce viable $(\mathbf{M})$ contralateral tumor tissue $(n=3)$. ${ }^{*} P<0.05,{ }^{* *} P<0.01,{ }^{* * *} P<0.0001, \dagger P<0.05$ compared with $37^{\circ} \mathrm{C}$ group, $\ddagger P<0.01$ compared with $37^{\circ} \mathrm{C}+$ Dox group. Scale bar: $3 \mathrm{~mm}$. Statistics for G-I were determined by ANOVA followed by Fisher's LSD without multiple comparisons correction, and those for J were determined by ANOVA followed by Tukey multiple comparison correction.

H\&E staining obtained at day 31 confirmed a large reduction in viable tumor cells within the treated tumor compared with tumors in nontreated control mice (Figure 1, K and L). All tumors treated with MRgFUS ablation contained residual tumor lesions in the periphery that were viable (Figure 1L, red arrows), presumably outside the path of the ultrasound beam and explaining the tumor regrowth seen in survival studies. The viability of contralateral tumors was unaffected by MRgFUS ablation (Figure 1M).

Thermal ablation alters the local tumor microenvironment and intratumoral transport. We then applied positron emission tomography (PET), contrast-enhanced T1-weighted MRI (CET1wMRI), and tumor histology to characterize the effects of MRgFUS ablation on solid tumor transport. ${ }^{64} \mathrm{Cu}$-labeled albumin was injected into the tail vein immediately after MRgFUS ablation of a single tumor in mice bearing bilateral 

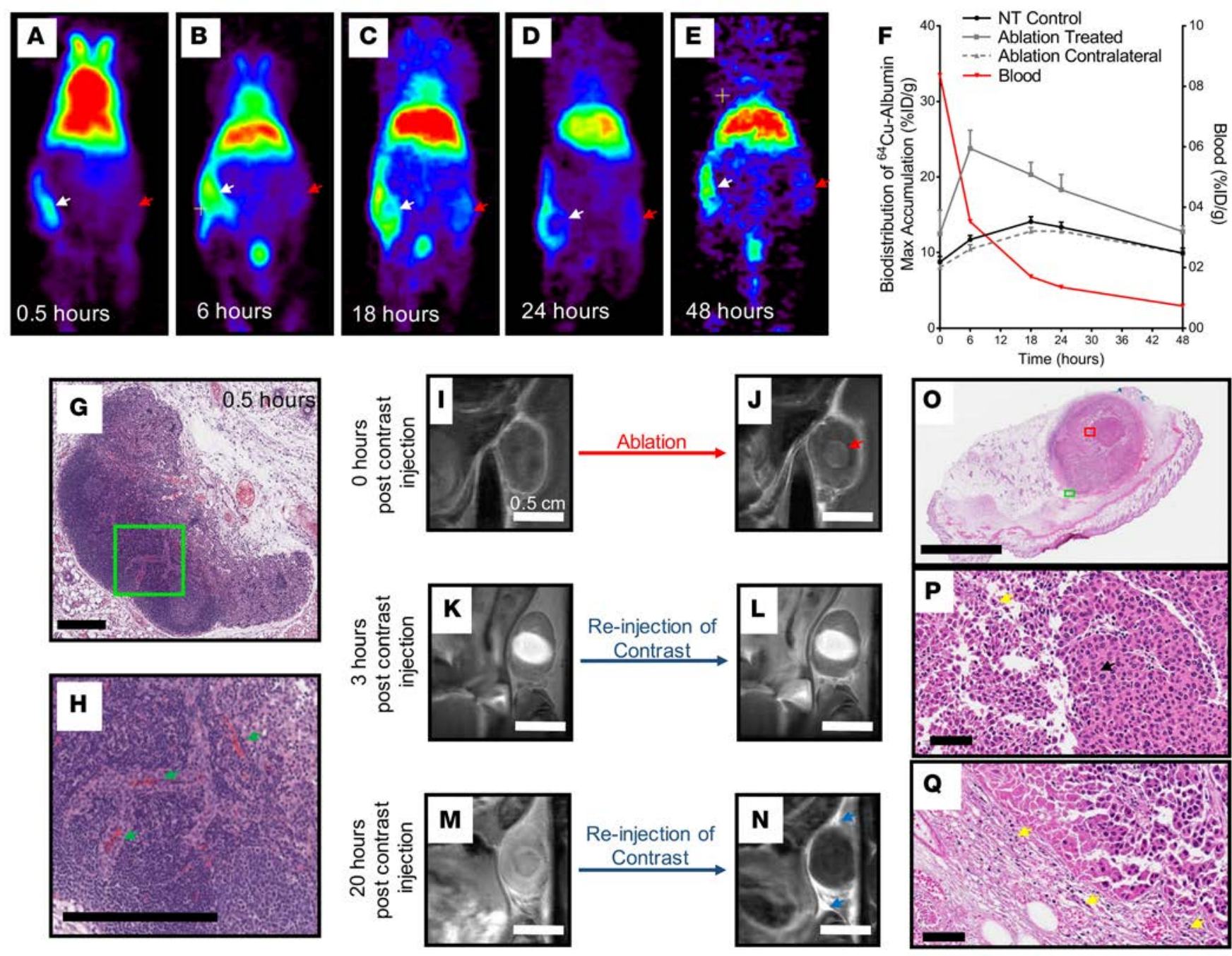

Figure 2. Thermal ablation alters the tumor microenvironment and intratumoral transport kinetics of small molecules and proteins. (A-F) Tumor permeability of proteins after thermal ablation was assessed in vivo by tracking Copper-64-labeled BSA ( $\left.{ }^{64} \mathrm{Cu}-\mathrm{BSA}\right)$ with positron emission tomography at (A) 0.5, (B) 6, (C) 18, (D) 24, and (E) 48 hours. A single tumor in a bilateral NDL tumor-bearing mouse was ablated (white arrow). Also visible are contralateral tumors (red arrow). Tracer kinetics and biodistribution were plotted for the (F) maximum intratumoral accumulation of ${ }^{64}$ Cu-BSA versus time, revealing a temporal peak of the spatial mean and maximum occurring at 6 hours after injection ( $n=4$ per cohort, data are mean \pm SEM). (G and $\mathbf{H})$ Increased vascularization of the tumor-draining lymph node 0.5 hours after tumor ablation upon H\&E histological staining $(n=3)$. Scale bar: $300 \mu \mathrm{m}$. (G) Draining lymph node following thermal ablation with green box at location of interest. $(\mathbf{H})$ View of enhanced vascularization within the lymph node indicated by green arrows. (I-N) Tumor permeability to small molecules after thermal ablation was assessed in vivo with contrast enhanced T1-weighted magnetic resonance imaging (CET1WMRI) ( $n=3$ ). (I) Gadoteridol was administered immediately before (J) ablation (red arrow). (K) Gadoteridol accumulated in the ablated region 3 hours after injection and ablation. (L) Contrast was readministered at 3 hours; additional accumulation was not detected. (M) At 20 hours after injection and ablation, intratumoral gadoteridol accumulation had cleared. (N) After 20 hours, gadoteridol readministration resulted in accumulation in the surrounding tumor rim (blue arrows) but not within in the ablated tissue. (0-Q) Mechanisms for enhanced accumulation 48 hours after thermal ablation with $\mathrm{H} \& \mathrm{E}(n=8)$. (0) Representative tumor following ablation with green and red box at locations of interest. Scale bar: $3 \mathrm{~mm}$. (P) Image from red box of heat-fixed tissue with shrunken but intact nuclei and preservation of tissue architecture (black arrow) surrounded by discohesive and nonviable tumor tissue with some ghosted nuclei and local edema (yellow arrow). Scale bar: $60 \mu \mathrm{m}$. (Q) Image from green box of inflammation observed, where leukocytes are densely located in the periphery of the tumor (yellow arrows). Scale bar: $60 \mu \mathrm{m}$.

tumors and traced over a 48-hour period (Figure 2, A-F). The ${ }^{64} \mathrm{Cu}$-labeled tracer accumulated in the tumor by 0.5 hours (Figure $2 \mathrm{~A}$ ), and the temporal peak of the spatial maximum accumulation occurred at 6 hours after MRgFUS ablation (Figure $2 \mathrm{~F}$ ). Moreover, it was evident that ${ }^{64} \mathrm{Cu}$-labeled albumin permeated away from the treated tumor over a period of 48 hours (Figure 2, B-F), suggesting enhanced lymphatic drainage. By 24 hours, ${ }^{64} \mathrm{Cu}$-labeled albumin accumulated only in the rim of the tumor (Figure $2 \mathrm{D}$ ) and was no longer evident in the center of the lesion. Immediately after ablation, H\&E of the lymph nodes near the ablation site demonstrated the presence of red blood cells within lymphatic channels, indicating enhanced 
permeability and transport in the tumor-draining lymph nodes (TDLNs, Figure 2, G and H, green arrows). To further elucidate changes in tumor vascular permeability, we assessed the accumulation of a small molecule, gadoteridol, in tumors with CET1wMRI after MRgFUS ablation (Figure 2, I-N). Gadoteridol was administered i.p. immediately before MRgFUS tumor ablation (Figure 2, I and J) and accumulated in the treated tumor within 3 hours after ablation (Figure $2 \mathrm{~K}$ ). Gadoteridol was readministered at the 3-hour time point (Figure 2L); however, additional accumulation was not significant. Similarly, 20 hours after ablation, gadoteridol reinjection did not enhance the ablated region or immediate surroundings (Figure 2, $\mathrm{M}$ and $\mathrm{N}$ ). Instead, gadoteridol accumulation was enhanced only in the rim of the tumor (Figure $2 \mathrm{~N}$, blue arrows) after reinjection of the contrast agent.

Tumor histology by H\&E demonstrated the biologic response to MRgFUS ablation (Figure 2, O-Q). The center of the tumor, where ablation was performed, appears heat fixed with shrunken but intact nuclei and with preservation of tissue architecture (Figure 2P, black arrow). Adjacent to this heat-fixed region, the surrounding tumor tissue is discohesive and nonviable, with some ghosted nuclei and local edema (Figure 2P, yellow arrow). Thus, our results suggest that, although there is increased vascular permeability and intratumoral (i.t.) transport immediately after FUS, this effect is short lived; within 24 hours, the central ablated region becomes impermeable, and transport of small and large tracers from the tumor is evident. Inflammation was observed 48 hours after tumor ablation, where leukocytes were densely concentrated in the periphery of the tumor (Figure 2Q, yellow arrows).

Development of the immunotherapy protocol: $C p G$ administration and $P D-1$ expression. PD-1 was expressed on $\mathrm{T}$ cells in bilateral tumor-bearing mice by day 21 (the time at which NDL tumors reached approximately $5 \mathrm{~mm}$ ) (Supplemental Figure 1, A-C; supplemental material available online with this article; https://doi. org/10.1172/jci.insight.90521DS1). Expression was greatest within the tumor $\left(4.8 \%\right.$ and $11.5 \%$ of $\mathrm{CD}^{+}$ T cells expressed CD 4 and PD-1 or CD8 and PD-1, respectively) and reduced within the tumor draining lymph node (1.4\% and $0.5 \%$ of CD3 ${ }^{+} \mathrm{T}$ cells expressed CD4 and PD-1 or CD8 and PD-1, respectively) and spleen ( $1.4 \%$ and $0.3 \%$ of $\mathrm{CD}^{+} \mathrm{T}$ cells expressed $\mathrm{CD} 4$ and $\mathrm{PD}-1$ or $\mathrm{CD} 8$ and PD-1, respectively). Given the known efficacy of PD-1 therapies and its expression in this model, $\alpha$ PD-1 therapy was included in subsequent protocols and combined with thermal ablation.

With CpG administered i.t., tumor growth was slowed within the treated tumor to a greater degree than within the contralateral tumor. The response, however, was heterogeneous as shown by the relatively early time point (46 days) at which the first CpG-treated tumor exceeded humane guidelines and the CpG recording was ended (Supplemental Figure 2). Adding ablation to $\mathrm{CpG}$ treatment did not further reduce the growth of the contralateral tumor on average. No member of the ablation $+\mathrm{CpG}$ cohort reached the maximum tumor volume $\left(1.5 \mathrm{~cm}^{3}\right)$ until day 56 with the combined treatment, as compared with day 46 for $\mathrm{CpG}$ treatment alone (Supplemental Figure 2, A and B). Further, treatment with $\alpha$ PD-1 only had no discernable effect on tumor growth compared with the no-treatment control (Supplemental Figure 2, C and D).

Immunotherapy begun coincident with thermal ablation diminished the efficacy of immunotherapy. We next evaluated the therapeutic efficacy of administering MRgFUS ablation immediately before (termed coincident with) immunotherapy. Since a protocol involving $\alpha$ PD-1 alone did not reduce tumor growth and $\mathrm{CpG}$ alone did not significantly reduce average contralateral tumor growth, we developed a protocol that coupled thermal dosing with checkpoint blockade of the PD-1/PD-L1 axis and TLR9 stimulation via $\alpha \mathrm{PD}-1$ and $\mathrm{CpG}$ administration, respectively (Figure 3A). To assess the efficacy of this coincident TA-immunotherapy approach, we investigated the effects of treatment on tumor growth, tumor histology, immunocyte populations, and animal survival in bilateral NDL tumor-bearing mice (Figure 3). We found that both immunotherapy alone and coincident TA-immunotherapy suppressed growth of the directly treated tumor (Figure 3B). While the inclusion of thermal ablation did not reduce the mean tumor growth rate beyond that achieved with immunotherapy alone, tumors treated with a thermal dose were observed to have more necrosis by day 35 (Supplemental Figure 3, A-D). This effect was transient, however, and tumor regrowth occurred in the majority of animals. Interestingly, contralateral tumor growth was significantly more rapid for coincident TA-immunotherapy as compared with immunotherapy-only protocols (Figure 3C). The mean tumor growth rate diverged near day 31 (after the second ablation) with significantly larger tumor growth for the ablation $+\mathrm{CpG}+\alpha \mathrm{PD}-1$ cohort after day 38 as compared with $\mathrm{CpG}+\alpha \mathrm{PD}-1$ treatment. Growth suppression was also reduced for ablation $+\mathrm{CpG}$, as compared with $\mathrm{CpG}$-treated animals, although the difference was not significant (Supplemental Figure 2B). Survival of all treatment groups was assessed over 100 or more days. The addition of $\mathrm{CpG}$ or $\mathrm{CpG}+\alpha \mathrm{PD}-1$ to MRgFUS ablation increased survival compared with MRgFUS 
A
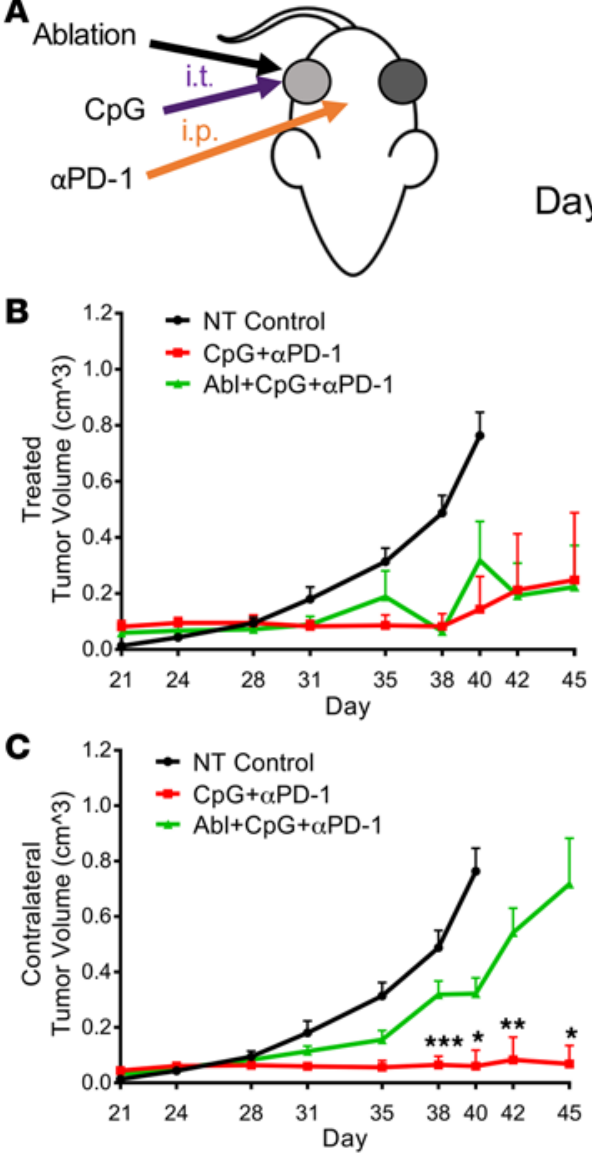

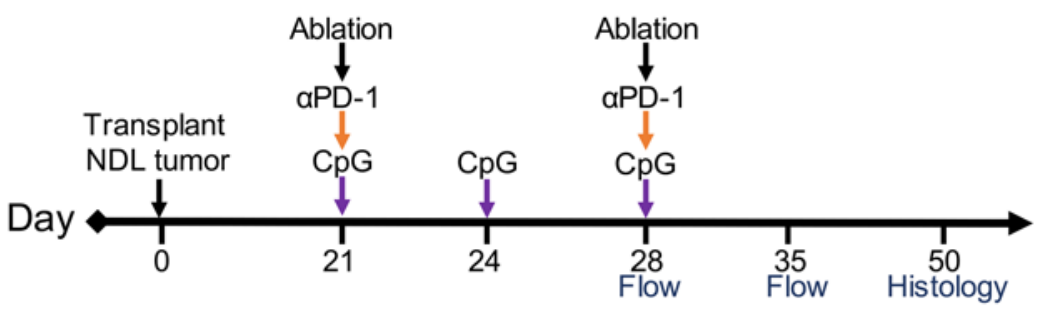

E

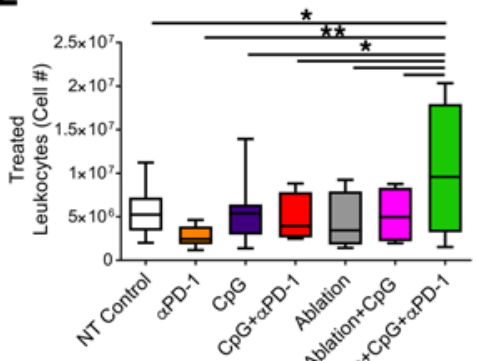

$\mathbf{F}$

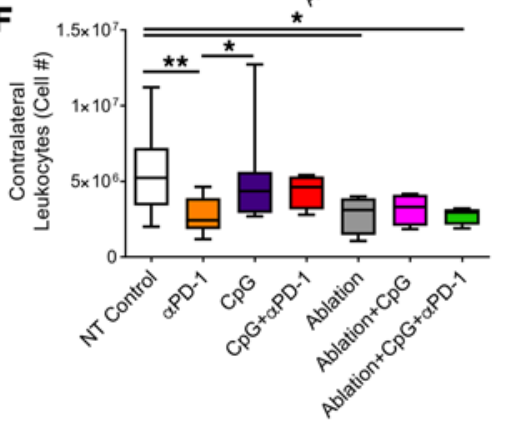

H
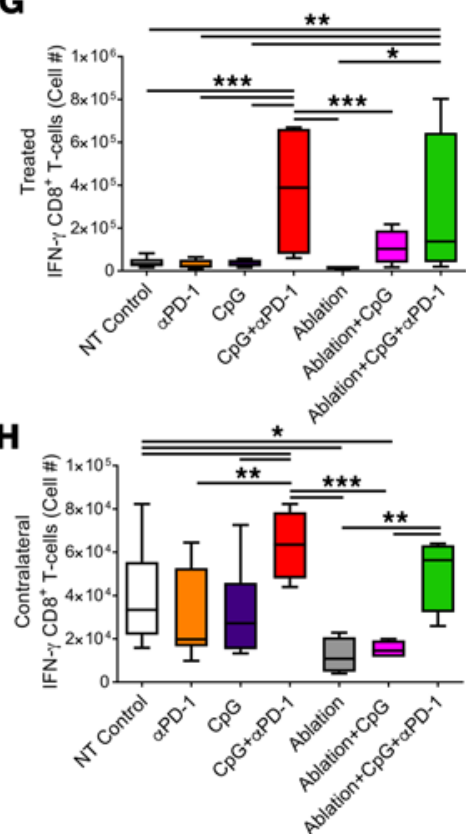

D

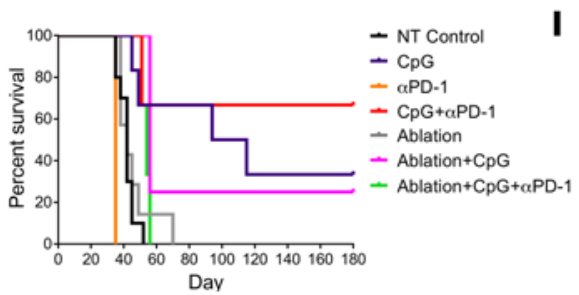

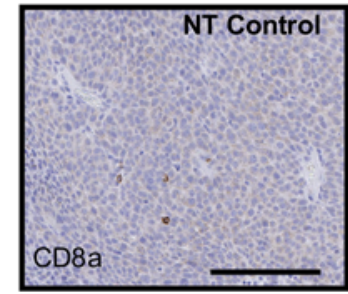

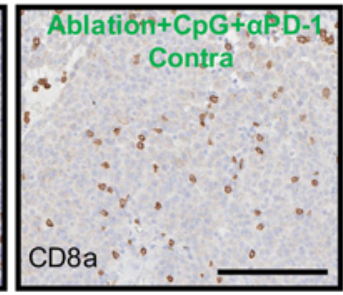

Figure 3. Coincident thermoablative immunotherapy protocol (TA-immunotherapy) diminishes the abscopal effects of immunotherapy. (A) Regimen of coincident TA-immunotherapy, CpG (100 $\mu$ g per injection, intratumoral [i.t.]) and anti-PD-1 ( $\alpha$ PD-1) (200 $\mu$ g per injection, i.p.) in mice orthotopically transplanted with NDL tumor biopsies in the fourth and ninth mammary fat pad. (B and C) Tumor growth was followed until an animal from the group was euthanized (tumor diameter $>1.5 \mathrm{~cm}$ ). Treatment cohorts were NT control $(n=8)$, CpG $+\alpha \mathrm{PD}-1(n=6)$ and ablation $+\mathrm{CpG}+\alpha \mathrm{PD}-1(\mathrm{Abl}+\mathrm{CpG}+\alpha \mathrm{PD}-1, n$ $=7)$. Tumor growth data result from survival study $(n=21)$ but are representative of trends in 7 studies with varied end points $(n=90$ total). (B) Immunotherapy alone and coincident TA-immunotherapy induced suppression of local tumor growth. (C) Contralateral tumor growth suppression was greater for immunotherapy alone. Data plotted as mean \pm SEM. (D) By day 180, survival outcomes for mice treated with immunotherapy alone (CpG $+\alpha P D-1, n=6)$ were improved compared with those treated coincident with ablation (ablation $+[p G+\alpha P D-1, n=7$ ) or control treatments (NT Control [ $n=8]$, $\alpha$ PD-1 [ $n=3$ ], CpG [ $n=7]$, ablation [ $n=7]$, and ablation + CpG $[n=4])$. $(\mathbf{E}-\mathbf{H})$ The fourth and ninth mammary fat pad (tumor and the embedded node) was harvested at day 28 , and immunocytes were quantified via flow cytometry $(n=4$ per group). Number of (E and $\mathbf{F})$ leukocytes and $(\mathbf{G}$ and $\mathbf{H})$ IFN- $\gamma$ CD8 $8^{+}$cells in treated ( $\mathbf{E}$ and $\mathbf{G}$ ) and contralateral $(\mathbf{F}$ and $\mathbf{H})$ tumors. For box-and-whiskers plots, the whiskers represent the minimum and maximum values, the box boundaries represent the 25th and 75th percentiles, and the middle line is the median value. (I) IHC on day 50 verified that both CpG $+\alpha P D-1(n=3)$ and ablation $+C p C$ $+\alpha \mathrm{PD}-1(n=3)$ increased infiltrating CD8 ${ }^{+} \mathrm{T}$ cells (brown stain) in contralateral tumors compared with NT controls. C was analyzed using an unpaired $t$ test assuming unequal variance comparing mean tumor volume of $\mathrm{CpG}+\alpha \mathrm{PD}-1$ and ablation $+\mathrm{CpG}+\alpha \mathrm{PD}-1$ at each day. E-H were analyzed by ANOVA followed by Fisher's LSD test without multiple comparisons correction. Scale bars: $150 \mu \mathrm{m} .{ }^{*} P<0.05,{ }^{* *} P<0.01,{ }^{* * *} P<0.001$.

ablation alone. Further, survival was extended in the immunotherapy-only cohort as compared with the coincident TA-immunotherapy cohort. $\mathrm{CpG}+\alpha \mathrm{PD}-1$ treatment produced a $66 \%$ survival rate at day 180 , and a complete response was achieved in all surviving animals (Figure 3D).

To understand the mechanisms underlying differences in responses of distant tumors to immunotherapy and coincident TA-immunotherapy, we evaluated immune cell populations with flow cytometry and IHC 
A

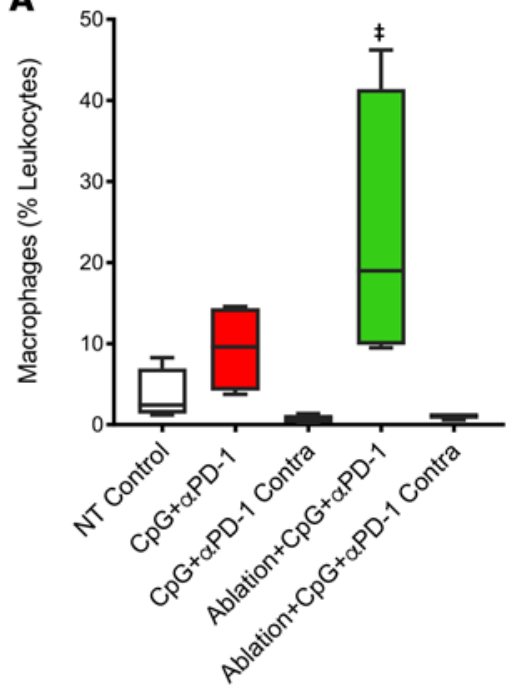

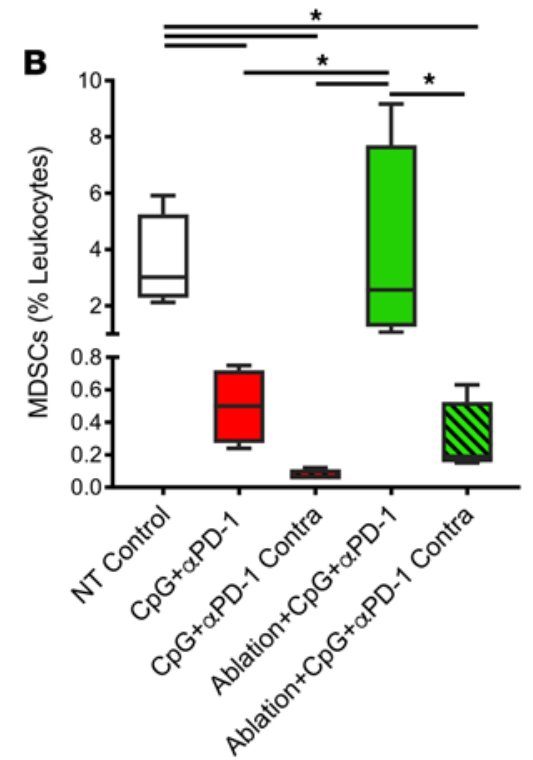

C

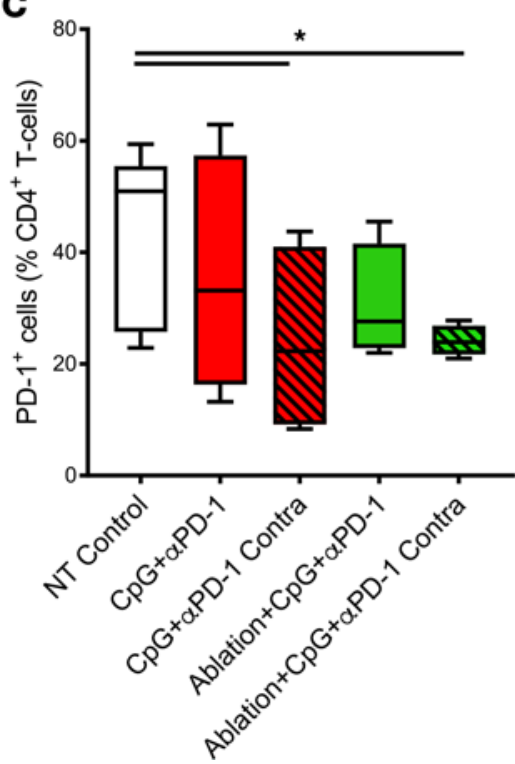

D

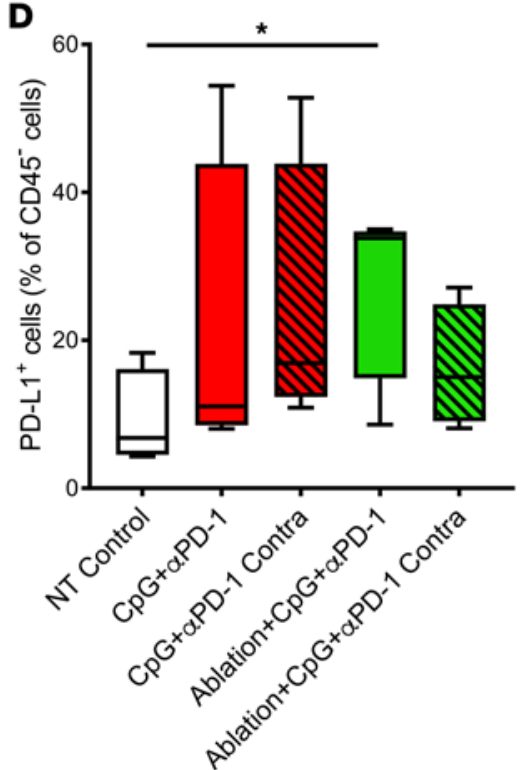

$\mathbf{E}$

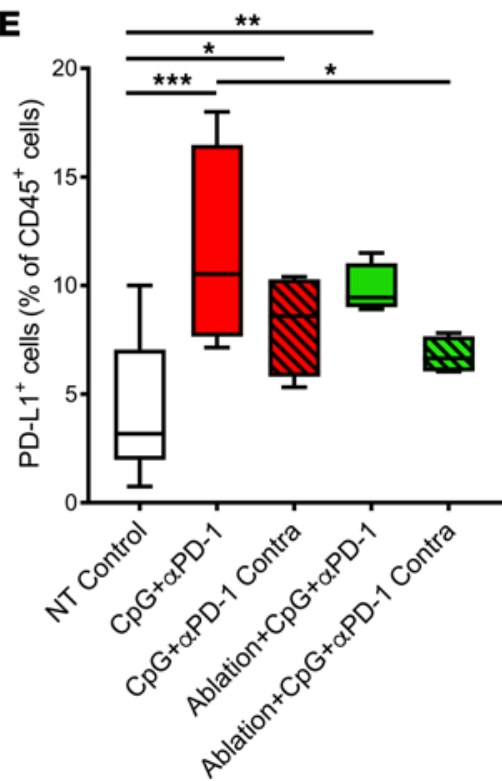

Figure 4. Comparison of coincident TA-immunotherapy (ablation $+C p G+\alpha P D-1)$ with immunotherapy alone (CpG $+\alpha P D-1)$. CpG $+\alpha P D-1$ reduces macrophages and myeloid-derived suppressor cells (MDSCs) in contralateral tumors and increases PD-L1 expression in tumor-infiltrating leukocytes. Coincident thermoablative immunotherapy (TA-immunotherapy) increases macrophages in treated tumors. (A-E) Animals were treated as described in Figure $3 A$ with thermal ablation, CpG (100 $\mu$ g per injection), and $\alpha$ PD-1 (200 $\mu$ g per injection). Treatment cohorts were NT control $(n=8)$, CpG $+\alpha \mathrm{PD}-1$ ( $n=8)$, and ablation $+C p G+\alpha P D-1(n=8)$. (A and B) The frequency of $(\mathbf{A})$ macrophages and (B) MDSCs in the treated and contralateral tumors following treatment. The fourth and ninth mammary fat pads (tumor and the embedded node) were harvested at day 35 and immunocytes were quantified via flow cytometry. (C-E) Expression of PD-1 and PD-L1 in treated and contralateral tumors. Tumors were harvested at day 28, and immunocytes were quantified via flow cytometry. (C) The amount of PD-1 expressed on CD4+ T cells, the (D) expression of PD-L1 on tumor/stromal cells (CD45-), and (E) the expression of PD-L1 on $\mathrm{CD}_{4} 5^{+}$leukocytes in treated and contralateral tumors. For box-and-whiskers plots, the whiskers represent the minimum and maximum values, the box boundaries represent the 25 th and 75 th percentiles, and the middle line is the median value. Statistics in A-E were determined by ANOVA followed by Fisher's LSD test without multiple comparisons correction. ${ }^{*} P<0.05,{ }^{* *} P<0.01,{ }^{* *} P<0.001, \ddagger P<0.05$ compared with all groups.

(Figure 3, E-I). T lymphocyte populations were similar following these treatments and the following components of these protocols ( $\alpha \mathrm{PD} 1, \mathrm{CpG}, \mathrm{CpG}+\alpha \mathrm{PD}-1$, ablation, ablation $+\mathrm{CpG}$, ablation $+\mathrm{CpG}+\alpha \mathrm{PD}-1)$, with a few exceptions (Figure 3 and Supplemental Figure 4). Coincident TA-immunotherapy enhanced lymphocytes within the treated tumors as compared with all other treatment groups (Figure 3E). Both immunotherapy and coincident TA-immunotherapy enhanced IFN- $\gamma$-producing $\mathrm{CD} 8^{+} \mathrm{T}$ cells in the treated and contralateral tumors compared with no-treatment controls when expressed as cell number or fraction of leukocytes (Figure 3, G and H, and Supplemental Figure 4, A-D). On IHC at day 50, infiltrating CD8 ${ }^{+}$ 
T cells (Figure 3I) were similarly elevated in ablation $+\mathrm{CpG}+\alpha \mathrm{PD}-1$ and $\mathrm{CpG}+\alpha \mathrm{PD}-1$ contralateral tumors compared with no-treatment controls. Treg density was similar across all treatment groups in contralateral tumors and was spatially distributed along the tumor periphery (Supplemental Figure 5, A-F).

In order to further characterize potential mechanisms for the reduced efficacy of coincident TA-immunotherapy, the fraction of macrophages and MDSCs was evaluated in both the treated and contralateral tumors. In the coincident TA-immunotherapy directly treated tumor, macrophages were elevated compared with all other treatment groups (Figure 4A). In the contralateral tumors, both macrophages and MDSCs were reduced following $\mathrm{CpG}+\alpha \mathrm{PD}-1$ and ablation $+\mathrm{CpG}+\alpha \mathrm{PD}-1$ treatments, and $\mathrm{M} 1$ macrophage markers were significantly elevated (Figure 4, A and B, and Supplemental Figure 6, A and B). MDSCs (Figure 4B) were significantly reduced in $\mathrm{CpG}+\alpha \mathrm{PD}-1$-treated tumors but not in ablation $+\mathrm{CpG}$ $+\alpha \mathrm{PD}-1-$ treated tumors, as compared with controls.

The expression of PD-1 and PD-L1 was then assessed after 1 week of treatment (day 28). The fraction of $\mathrm{PD}-1$ expression on tumor-infiltrating $\mathrm{CD}^{+} \mathrm{T}$ cells was reduced in contralateral tumors of mice treated with ablation $+\mathrm{CpG}+\alpha \mathrm{PD}-1$ and $\mathrm{CpG}+\alpha \mathrm{PD}-1$ (Figure $4 \mathrm{C}$ ). In mice treated with coincident TA-immunotherapy, PD-L1 was enhanced on CD45- tumor and stromal cells in the treated tumor, and a trend toward enhancement was observed in other groups (Figure 4D). Further, PD-L1 expression was significantly elevated on $\mathrm{CD}_{4} 5^{+}$cells in treated tumors from both $\mathrm{CpG}+\alpha \mathrm{PD}-1$ and ablation $+\mathrm{CpG}+\alpha \mathrm{PD}-1$ groups (Figure $4 \mathrm{E})$. However, in the contralateral tumors, PD-L1 was significantly increased following immunotherapy but not following coincident TA-immunotherapy (Figure 4E). Given this combination of changes in immune phenotype resulting from $\mathrm{CpG}+\alpha \mathrm{PD}-1$ (enhanced IFN- $\gamma$-producing $\mathrm{CD} 8^{+} \mathrm{T}$ cells, reduced MDSCs and macrophages, an enhanced M1 macrophage fraction in distant tumors, and enhanced PD-L1 expression in CD $45^{+}$cells), a 7-day course of $\mathrm{CpG}+\alpha \mathrm{PD}-1$ was next evaluated as a priming protocol before ablation. Further, these results provide evidence of both reduced antitumor activity and lower efficiency in modulating the tumor-suppressive environment with a coincident TA-immunotherapy protocol.

Priming prior to thermal ablation rescues efficacy and generates a rapid antitumor response. We next investigated sequential administration of 7 days of immunotherapy followed by a TA-immunotherapy protocol (primed TA-immunotherapy) or continued immunotherapy alone (Figure 5A). We found that primed TA-immunotherapy (ablation $+\mathrm{CpG}+\alpha \mathrm{PD}$-1-Prime) and immunotherapy (CpG $+\alpha \mathrm{PD}-1$ Prime) treatments dramatically suppressed tumor growth in both the treated and contralateral tumors, producing an $80 \%$ complete response by day 90 (Figure 5, B and C). Tumor growth suppression occurred sooner for primed TA-immunotherapy compared with immunotherapy alone (Figure 5, B and $\mathrm{C})$. In particular, for the $\mathrm{CpG}+\alpha \mathrm{PD}$-1-Prime cohort, contralateral tumor volume increased in all mice up to day 38 , reaching a peak of $\sim 0.15 \mathrm{~cm}^{3}$, while peak volume was $\sim 0.06 \mathrm{~cm}^{3}$ for the treatment incorporating ablation. For this intense immunotherapy protocol ( 6 injections of $\mathrm{CpG}$ and 3 injections of $\alpha \mathrm{PD}-1)$, the primed groups yielded the same survival outcome in bilateral tumors with and without ablation (Figure 5D). However, the rapid response achieved by adding ablation suggests that the immunotherapy dose could be reduced in the future.

Through H\&E staining at an early time point (day 35), we found that tumor cell death was more homogeneous with the primed TA-immunotherapy than with immunotherapy alone (Supplemental Figure 7, A-J). Ablation $+\mathrm{CpG}+\alpha \mathrm{PD}-1$-Prime treatment induced tumor cell death throughout the ablated lesion, as indicated by necrosis, ghosted, and pyknotic nuclei (Supplemental Figure 7, C-D). Similarly, contralateral tumor cell death was striking with the ablation $+\mathrm{CpG}+\alpha \mathrm{PD}-1$-Prime treatment, including i.t. hemorrhage and loss of cell-cell adhesion (Supplemental Figure 7, E and F). Response in $\mathrm{CpG}+\alpha \mathrm{PD}$-1-Prime-treated and contralateral tumors was heterogeneous at this time point; viable tumor tissue existed throughout the treated (Supplemental Figure 7, G and H) and contralateral lesions (Supplemental Figure 7, I and J).

To characterize this rapid immune response, we assessed immunocyte infiltration into treated and contralateral tumors at day 35 via flow cytometry. Ablation $+\mathrm{CpG}+\alpha \mathrm{PD}-1$-Prime treatment significantly increased leukocyte (Figure $5 \mathrm{E}$ ), $\mathrm{CD}^{+} \mathrm{T}$ cell (Figure $5 \mathrm{~F}$ ), CD4 ${ }^{+} \mathrm{T}$ cell (Figure $5 \mathrm{G}$ ), and $\mathrm{CD} 8^{+}$ $\mathrm{T}$ cell (Figure $5 \mathrm{H}$ ) frequencies in the contralateral tumors compared with no-treatment controls. Compared with the $\mathrm{CpG}+\alpha \mathrm{PD}-1$-Prime treatment, leukocytes (Figure $5 \mathrm{E}$ ) and $\mathrm{CD} 8^{+} \mathrm{T}$ cells (Figure $5 \mathrm{H}$ ) in ablation $+\mathrm{CpG}+\alpha \mathrm{PD}$-1-Prime-treated mice were also significantly increased in contralateral tumors. IFN- $\gamma$-producing $\mathrm{CD}^{+} \mathrm{T}$ cells were significantly increased within the spleen when $\mathrm{CpG}$ and $\alpha \mathrm{PD}-1$ were combined with ablation (Supplemental Figure 8), further indicating enhanced systemic effect. 
A

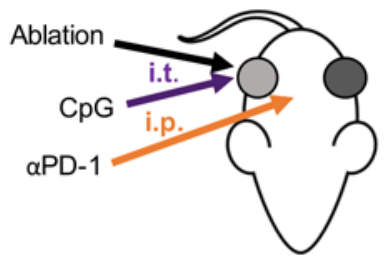

B
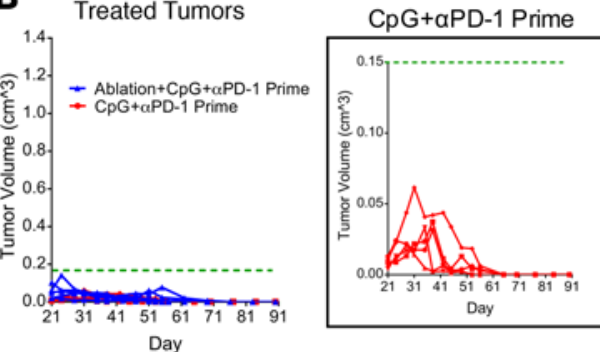

Day
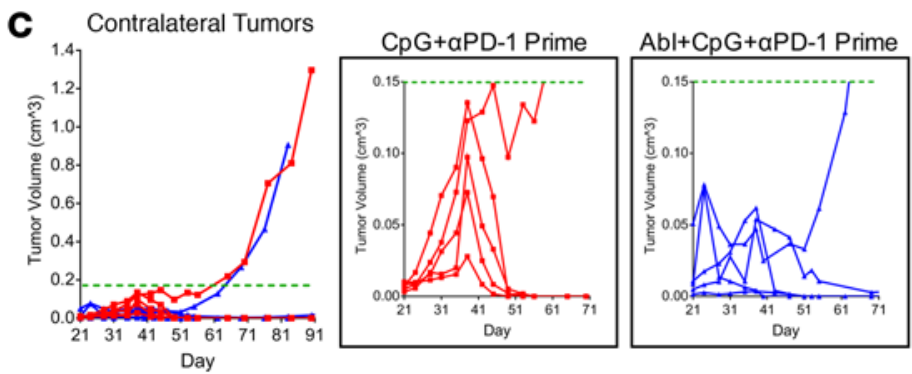

D
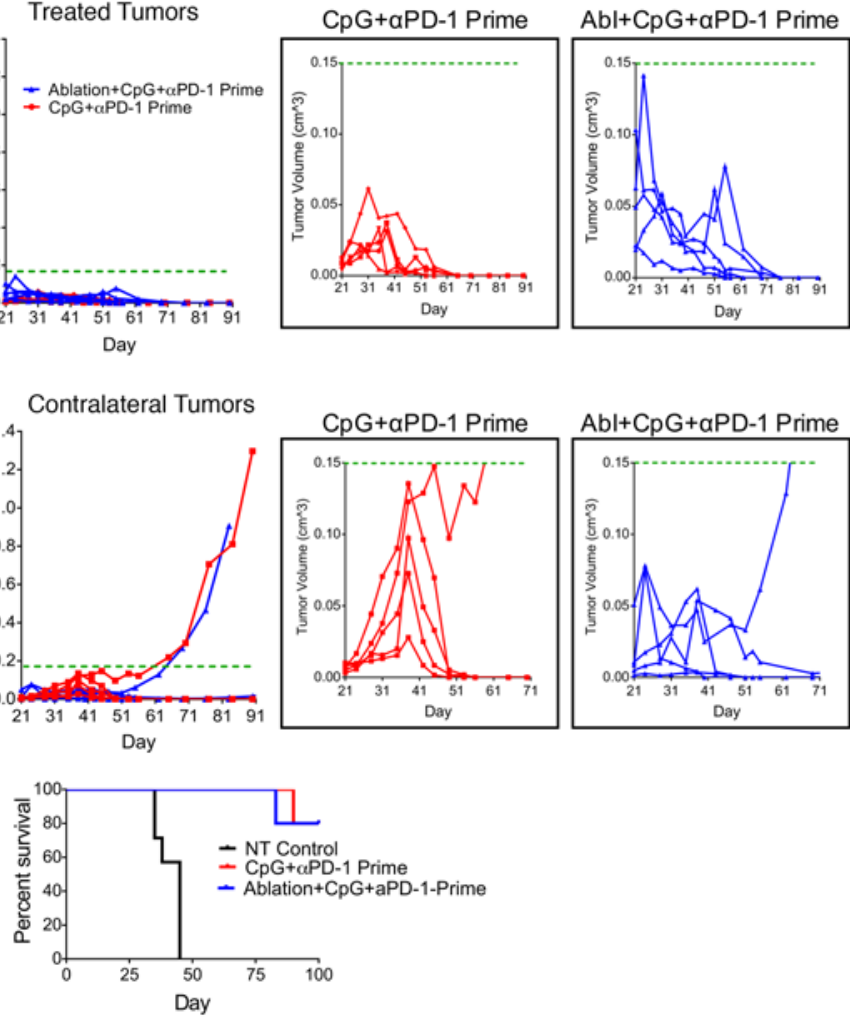

Priming protocol

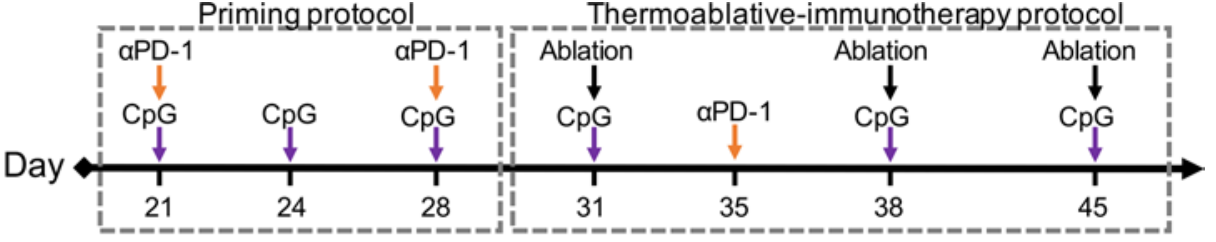

E

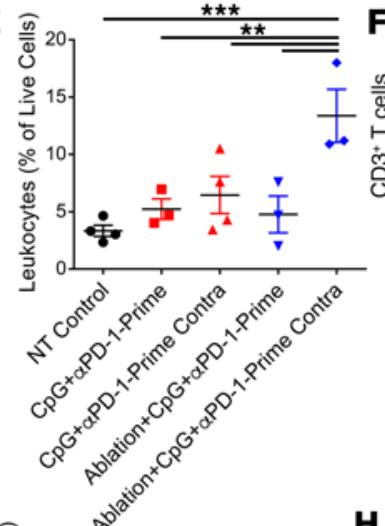

$\mathbf{F}{ }^{100} 1$
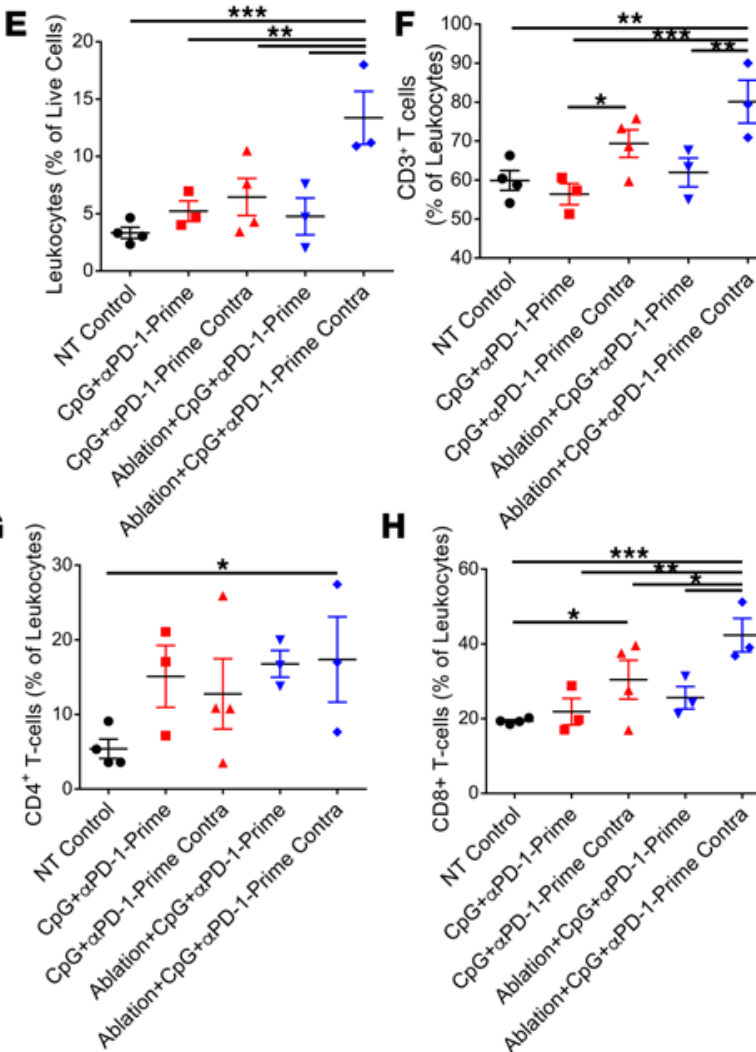

$\mathbf{H}$

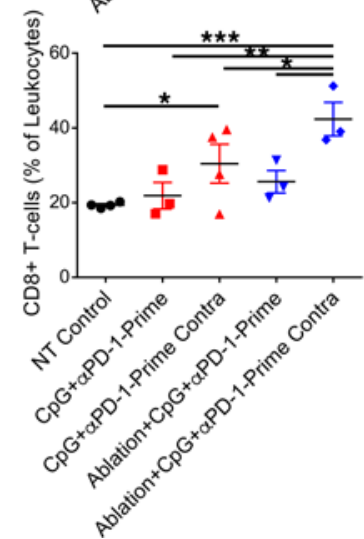

Figure 5. Priming the immune system is required for efficacious incorporation of thermal ablation into an immunotherapeutic protocol. (A) Regimen of primed thermoablative immunotherapy (TA-immunotherapy). Immunotherapy was administered prior to thermal ablation in priming protocol. Following priming, mice received a combination of thermal dosing and immunotherapy in TA-immunotherapy protocol. Treatments included $\mathrm{CpC}$ $+\alpha$ PD-1-Prime $(n=5)$, ablation + CpG $+\alpha$ PD-1-Prime (Abl + CpG $+\alpha$ PD-1-Prime, $n=5)$, and NT control $(n=4)$. CpC was injected intratumorally (i.t., 100 $\mu \mathrm{g})$, and $\alpha \mathrm{PD}-1$ was injected i.p. $(200 \mu \mathrm{g})$. Tumor growth data result from survival study $(n=14)$, but trends are representative of 4 separate experiments with varied end points (total $n=50$ ). (B and $\mathbf{C}$ ) $C p G+\alpha P D-1-P r i m e$ and ablation $+C p G+\alpha P D-1$-Prime achieved an $80 \%$ complete response by day $90(n=5)$. (B) $100 \%$ of all treated tumors and (C) $80 \%$ (4 of 5) of all contralateral tumors from both groups were eradicated after treatment; enhanced views (tumor volume plotted below dotted green line) of the treated (B) and contralateral (C) tumor growth from both treatment groups. (D) Survival outcomes for mice treated with the priming protocol (CpG $+\alpha \mathrm{PD}-1$-Prime, ablation $+C p G+\alpha P D-1-P r i m e$, and NT Control, $n=4$ per group). (E-H) On day 35, tumors were harvested, and leukocytes and T cells were quantified via flow cytometry (data are plotted as mean \pm SEM, $n=4$ per group). Effect of therapy on (E) CD45 leukocytes, (F) $C D 3^{+} T$ cells, (C) $C D 4^{+} T$ cells, and $(\mathbf{H}) C D 8^{+} T$ cells. Significance was determined via 1-way ANOVA followed by a Fisher's LSD test without multiple comparisons correction. ${ }^{*} P<0.05,{ }^{* *} P<0.01,{ }^{* *} P<0.001$.

In a direct comparison with other protocols incorporating ablation, primed TA-immunotherapy dramatically reduced tumor growth; however, for bilateral tumors, primed immunotherapy alone reduced tumor growth to a similar extent as primed TA-immunotherapy (Figure 6A). Further, tumor-infiltrating $\mathrm{CD}^{+} \mathrm{T}$ cells in the contralateral tumor were dramatically increased by primed TA-immunotherapy (Figure 6B), as compared with control groups including ablation (Figure 6C), ablation + CpG (Figure 6D), coincident TA-immunotherapy (Figure $6 \mathrm{E}$ ), and $\mathrm{CpG}+\alpha \mathrm{PD}-1$-Prime (Figure $6 \mathrm{~F}$ ).

Priming and thermal ablation of multiple tumors generates a rapid, complete response in distant lesions. We found that primed TA-immunotherapy resulted in a complex local microenvironment by day 38 (Figure 7, A-C), with dying tumor and inflammation (Figure 7B) surrounding a central pocket of collagen-dense and tumor-free stroma (Figure 7C). This complex environment generated concern for diffusion of subsequently injected material and guidance of reablation of remaining tumor. We therefore evaluated the 
A

Contralateral Tumor Growth
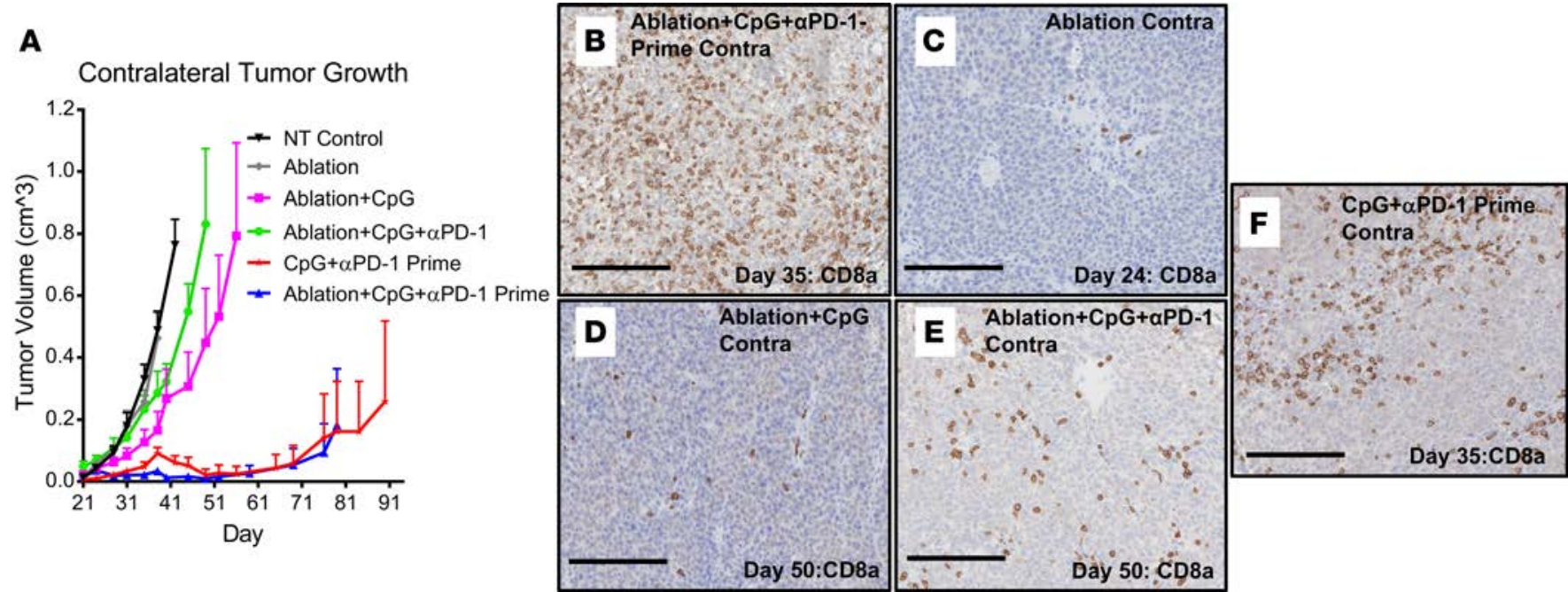

Figure 6. Direct comparison of results from primed thermoablative immunotherapy (TA-immunotherapy), coincident TA-immunotherapy, and immunotherapy alone demonstrates enhanced response. (A) Priming prior to thermal ablation (ablation + CpG $+\alpha P D-1-P r i m e ; ~ n=5$ ) suppressed contralateral tumor growth compared with other treatments that incorporated ablation, including NT control $(n=8)$, ablation $(n=7)$, ablation + CpG $(n=4)$, and ablation + CpG $+\alpha$ PD-1 $(n=7)$. For bilateral tumors, the growth of primed TA-immunotherapy was similar to primed immunotherapy alone $(n=5)$. Data are plotted as mean \pm SEM. (B-F) Infiltrating CD8 ${ }^{+}$T cells (brown stain) in contralateral tumors were increased by $(\mathbf{B})$ primed TA-immunotherapy as compared with (C) ablation, (D) ablation $+\mathbf{C p G},(\mathbf{E})$ coincident TA-immunotherapy, and $(\mathbf{F})$ primed immunotherapy $(n=3$ per group) upon immunohistochemical staining of CD8. Scale bars: $150 \mu \mathrm{m}$.

primed TA-immunotherapy protocol in an animal model with multiple tumor sites where 2 sites could be sequentially treated (Figure 7, D and E). To accomplish this, 3 NDL tumor biopsies were implanted, of which 2 were directly treated with ablation and/or immunotherapy. The abscopal effect was enhanced by multisite treatment; $80 \%$ of the nontreated tumors in the primed TA-immunotherapy were eliminated by day 56 compared with $25 \%$ in the immunotherapy-only group (Figure $7 \mathrm{~F}$ ). By day 35, tumor volume in nontreated (Figure 7F) and primed TA-immunotherapy-treated tumors (Figure 7G and Supplemental Figure 9A) was significantly lower than the control cohort $(P<0.05)$. In TA-immunotherapy-treated mice at day 50, tumor growth was suppressed in nontreated tumors to a greater extent than with immunotherapy only (Supplemental Figure 9B). Survival was enhanced by the sequential primed TA-immunotherapy, with $60 \%$ and $25 \%$ of mice treated with primed TA-immunotherapy and immunotherapy-only surviving at day 100 , respectively (Figure $7 \mathrm{H})$.

\section{Discussion}

This is the first report of the efficacy of combining ultrasound-mediated ablation with checkpoint immunotherapy protocols. Our goal was to determine whether such focal therapy can be effectively incorporated within an immunotherapy regimen, given the clinical significance of such focal therapies. Local treatments including radiation and surgery have each been shown to have both tumor-suppressive and tumor-promoting effects; a similar paradigm is seen here $(9,14,28)$.

Most importantly, our data demonstrate that optimizing the timing of immunotherapy and thermal ablation is crucial for the creation of a curative therapy. We found that a 7-day immunotherapy priming protocol depressed macrophages and MDSCs as a fraction of leukocytes, increased the fraction of M1 macrophages in distant tumors, and enhanced PD-L1 expression and IFN- $\gamma$ CD $8^{+} \mathrm{T}$ cells in both the treated and contralateral tumors. Ablation following this immunotherapy protocol (primed TA-immunotherapy) was then applied in an environment that was skewed toward an antitumor protocol, resulting in increases in leukocytes and $\mathrm{CD}^{+} \mathrm{T}$ cells, as compared with all control groups. With this primed TA-immunotherapy protocol, the abscopal effects of $\mathrm{CpG}$ and $\alpha \mathrm{PD}-1$ resulted in the complete response of $80 \%$ of treated mice with bilateral disease at day 90 . For bilateral tumors, the survival of mice treated with the primed TA-immunotherapy protocol was similar to that achieved for immunotherapy alone. A greater impact on survival was achieved by the primed TA-immunotherapy protocol when more than 2 lesions were present and were successively treated (2 of 3 lesions). A 60\% sustained complete response 

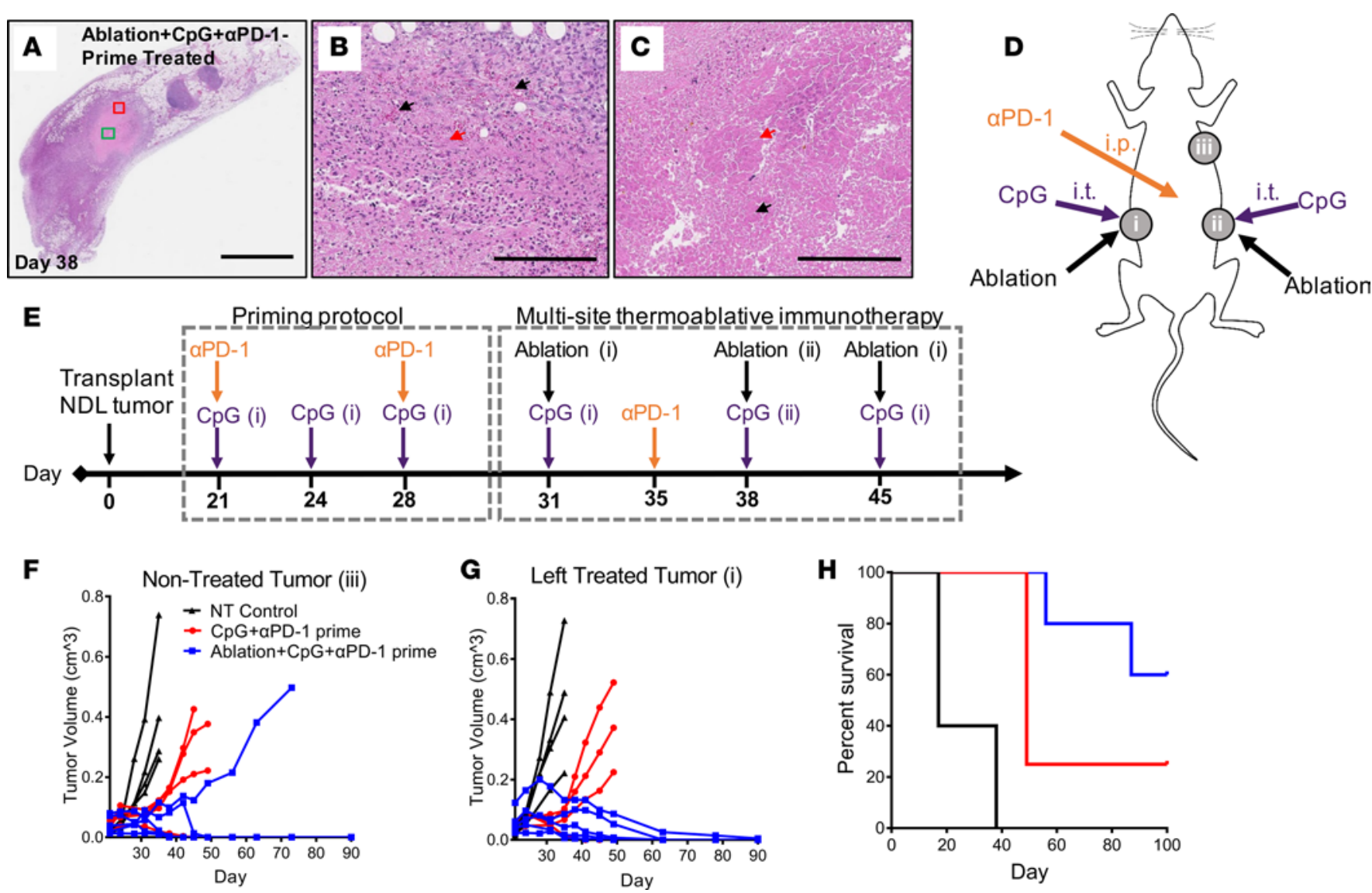

Multi-sitę ther
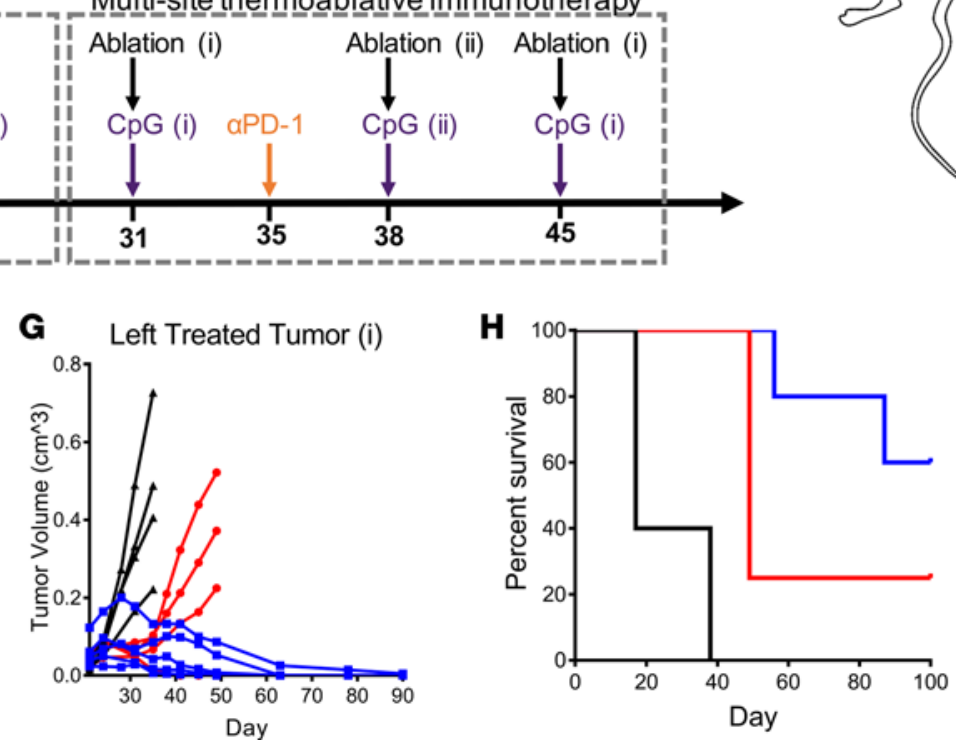

Figure 7. Sequential multisite thermoablative immunotherapy (TA-immunotherapy) enhances response. (A-C) Treatment with single-site TA-immunotherapy (ablation $+C p G+\alpha P D-1-P r i m e)$ results in heterogeneous lesions by day $38(n=4)$. (A) Histological section of ablation + CpG $+\alpha P D-1-P r i m e-t r e a t e d$ tumor with boxes at regions of interest. Scale bar: $4 \mathrm{~mm}$. (B) Enlarged view of red box, with local inflammation (infiltration of leukocytes), hemorrhage (black arrows), and necrotic tumor cells containing ghosted nuclei (red arrow). Scale bar: $200 \mu \mathrm{m}$. (C) Enlarged view of green box with heat fixation (red arrow), tumor necrosis, and collagen remodeling (black arrow). Scale bar: $200 \mu \mathrm{m}$. (D-H) Mice bearing 3 NDL tumors were treated with priming and multisite ablation. Tumor growth was followed until an animal from the group was euthanized (tumor diameter $>1.5 \mathrm{~cm}$ ). Treatment cohorts were NT control $(n=4), C p G+\alpha P D-1-P r i m e(n=4)$, and ablation $+C p G+\alpha P D-1$-Prime $(n=5)$, where data are plotted as mean \pm SEM. Mice per group examined in 2 separate experiments. (D) Mice were orthotopically transplanted with NDL tumor biopsies in the ninth (i), fourth (ii), and second mammary fat pad (iii). (E) Regimen of multisite administration of ablation $+C p G+\alpha P D-1-P r i m e$. CpG was injected intratumorally (i.t., $100 \mu \mathrm{g}$ ), and $\alpha$ PD-1 was injected i.p. (200 $\mu \mathrm{g})$. (F) Effect of multisite administration on tumor growth in nontreated (iii) and (C) left-treated (i) tumors. (H) Survival outcomes for mice treated with the multisite priming protocol $\mathrm{CpG}+\alpha \mathrm{PD}-1$-Prime $(n=4)$, ablation $+\mathrm{CpG}+\alpha \mathrm{PD}-1$-Prime $(n=5)$, and NT Control $(n=4)$.

was achieved with this sequential multisite treatment as compared with a $25 \%$ response achieved with immunotherapy alone. In comparison with distant tumors in a bilateral NDL-bearing mouse receiving the single-tumor treatment protocol, the abscopal response was more rapid. The evaluation of multifocal tumor treatment was motivated by the clinical significance of the treatment of disseminated disease, the recognition that immunotherapy efficacy is compromised in patients with a large tumor burden $(29,30)$, and the observation of changes in the tumor microenvironment produced by ablation. Based on these observed changes, we hypothesized that sequential treatment of more than one site could be more effective. We found that the response to immunotherapy degraded with enhanced tumor burden (as in refs. 29,30 ), whereas ablation reduced this tumor burden and improved response.

For the coincident TA-immunotherapy protocol, the abscopal effects were diminished as compared with immunotherapy alone or primed TA-immunotherapy, and none of the treated mice exhibited a complete response. This again underscores the importance of the protocol and timing. Two mechanisms have the potential to impact the efficacy of immunotherapy when begun after ablation: mechanical changes in the tumor microenvironment and inflammatory-mediated changes in immune phenotype. Rapid tumor cell death, destruction of blood vessels, and loss of cell-cell adhesion were observed within the ablated region. The rapid cell death resulted in heat fixation of central tumor tissue immediately after treatment, suggesting 
that a portion of the tumor is incapable of providing the immune system with viable antigen. The kinetics of i.t. transport of small molecules and proteins were greatly altered after treatment. As we have shown previously in ref. 25 , a small molecule contrast agent accumulated within the ablated area with the concentration peaking at 1.5 hours after treatment. Here, we show that reinjection of the contrast agent at later time points does not result in accumulation. Further, PET tracing of a systemically circulating protein confirmed accumulation in the ablated tumor peaked at $\sim 6$ hours after treatment before approaching the baseline level within 48 hours. Thus, locally injected immunotherapy, such as the TLR9 agonist applied here, is expected to at least partially clear from the ablated site, and systemically injected immunotherapy will reach the ablated rim but will largely be excluded from the center of the lesion. These changes in microenvironment and perfusion at each tumor site after ablation motivated the successive ablation of multiple lesions.

The second potential issue in the incorporation of focal therapy within immunotherapy protocols is the generation of a local inflammatory response. After a large inflammatory insult, MDSC proliferation and M2 polarization are enhanced, and such changes can be harmful to the antitumor immune response, an effect termed rebound immune suppression (31). M2 macrophages are reported to limit the response to radiation (28); a large increase in macrophages and MDSCs and a trend toward the M2 phenotype were observed following ablation concurrent with immunotherapy. Alternatively, the priming protocol (CpG $+\alpha \mathrm{PD}-1)$ generates a robust antitumor immune response and mitigates the inflammatory insult caused by focal therapy, and therefore the combination of the priming protocol and thermal ablation can be very effective.

The optimal dosing and timing regimen for combined immunotherapy and radiotherapy has previously been reported to vary (14). While fractionated radiotherapy has been reported to synergize with antiCTLA-4 therapy (32) or with a TLR7 agonist (33), other reports have indicated that concurrent treatment with $\alpha$ PD-1/PD-L1 or $\alpha$ PD-1 and anti-CD137 was effective (14). A similarly complex question exists for immunotherapy and ablation, but the timing of the response is very different. Thermally mediated cell death within the immediate region is rapid and can create an immediate source of antigen to enhance tumor recognition (34). A rapid response is attractive since clonal evolution can produce ever more aggressive phenotypes, which are resistant to targeted therapeutics $(35,36)$ and immunotherapeutic toxicity can be dose limiting (37).

Although we describe some disadvantages of thermal and focal therapies above, there are also significant advantages to incorporating these methods within immunotherapy protocols. As with radiotherapy, treatment-related release of factors associated with ICD, such as HMGB1 and local increases in IFN- $\gamma$, are associated with tumor rejection. As with surgery, tumor debulking is expected to eliminate a significant fraction of tumor-induced immunosuppression. When combining such treatments with immunotherapy, a hypothesized requirement is for some mass of immune-related factors, including tumor-associated antigens and $\mathrm{T}$ cells, to remain. With image-guided therapies, we can control the volume of tumor ablated or remaining in many instances. For thermal ablation, we chose a protocol in which the ablated region did not fully encompass the entire tumor, avoiding heat fixation of the tumor rim and providing a source of tumor antigen. Unlike many previous methods of thermal ablation, here the temperature is continuously monitored and controlled, and the treated region can be conformed to the disease site. Thermal ablation can be successfully applied regardless of the tumor environment; there is no limitation on its use in poorly perfused and hypoxic tumors.

It is important to recognize that the immune environment in this mouse model prior to treatment provided a suitable phenotype for immunotherapy. Immunotherapy, ablation, and combined protocols may each be less effective in tumor models with fewer native T cells and a greater fraction of MDSCs.

In summary, our data suggest that a primed ablation protocol can enhance treatment response with a high tumor burden. Also, as a result of mechanical and immunologic changes in the tumor microenvironment following from local ablation, there is a window of opportunity for employing immunotherapy, after which the ablation may actually limit the efficacy of immunotherapy. Given the growing use of both of these modalities in the clinic and the interest in combining focal therapies with immunotherapy, these findings have profound and direct clinical implications for the design of new treatment strategies for metastatic cancer.

\section{Methods}

Supplemental Methods are available online with this article.

Animal models. The NDL metastatic mammary carcinoma cell line was obtained from the Alexander Borowsky Laboratory (University of California, Davis) (27, 38). FVB/n mice (6-10 weeks old, 15-25 g, 
Charles River Laboratories) were orthotopically transplanted with syngeneic NDL tumor biopsies $(\sim 1$ $\mathrm{mm}^{3}$ ) into the bilateral \#4 and \#9 inguinal mammary fat pads as previously described (39). For studies involving multisite ablation, a third NDL tumor $\left(\sim 1 \mathrm{~mm}^{3}\right)$ was placed orthotopically into the \#2 mammary fat pad on the right side.

MRgFUS ablation protocol. MRgFUS was performed with a Bruker BioSpec 7T small animal system (Bruker Biospin), MR-compatible 16-element annular transducer (Imasonic SAS) (48 mm diameter, $35 \mathrm{~mm}$ radius of curvature, $3 \mathrm{MHz}$ central frequency), and MR-compatible transducer positioning system (Image Guided Therapy). The treatment plan used 5 acoustic Watts and an acoustic pressure of 3.1 MPa. Acoustic pressure was calibrated with a fiber optic hydrophone (HFO690, Onda Corp.) in a degassed water bath in free-field conditions. The highly focused annular array achieved a $0.5 \mathrm{~mm} \times 0.5 \mathrm{~mm} \times 1.5 \mathrm{~mm}$ instantaneous focal volume. The focal volume was scanned through the tumor volume in a circular pattern with the diameter selected to ablate to within 1-2 mm of the tumor edge and with a scan speed of 1 revolution per second. Thus, heat diffusion from the rim to the center facilitated ablation of the tumor volume. Each treatment plan was designed to induce temperatures greater than $65^{\circ} \mathrm{C}$ and a thermal dose in cumulative equivalent minutes at 43 degrees (CEM43) of more than 5,000.

Mice were first imaged with T1-weighted MRI for tumor localization, using the MRI images to position the mouse such that the ultrasound beam did not pass through sensitive organs. Scan parameters of echo time $/$ repetition time $(\mathrm{TE} / \mathrm{TR})=12.5 / 750 \mathrm{~ms}$, field of view $(\mathrm{FOV})=3.2 \mathrm{~cm} \times 3.2 \mathrm{~cm}$, acquisition matrix $(\mathrm{MTX})=256 \times 256$, slice thickness/slice interval $(\mathrm{ST} / \mathrm{SI})=1 / 1 \mathrm{~mm}$, and 9 slices were used for T1-weighted imaging. Temperature was monitored by the MR proton resonance frequency shift using Thermoguide software (Image Guided Therapy), with $\alpha=-0.0101 \mathrm{ppm} /{ }^{\circ} \mathrm{C}, \mathrm{TE} / \mathrm{TR}=4.5 / 21 \mathrm{~ms}$, as described (40). Temperature was validated with a Luxtron STB fiber optic probe (Lumasense Technologies). Prior to MRgFUS, mice were given $0.05-0.1 \mathrm{mg} / \mathrm{kg}$ buprenorphine s.c. and $0.05 \mathrm{mmol} / \mathrm{kg}$ of gadoteridol (Bracco Imaging) via i.p. injection.

Reagents. CpG-ODN 1826 (5'-tccatgacgttcctgacgtt-3'; total backbone phosphorothioated) was purchased from InvivoGen. The checkpoint inhibitor, rat anti-mouse PD-1 antibody ( $\alpha$ PD-1, RMP1-14) was purchased from Bio X Cell.

In vivo studies. Briefly, therapy was started when tumors reached approximately $0.5 \mathrm{~cm}$ in the largest diameter. A total of 210 NDL tumor-bearing mice were studied, randomized among groups including: no-treatment control $(n=40)$, ablation $(n=35)$, CpG $(n=17)$, $\alpha \mathrm{PD}-1(n=12)$, ablation $+\mathrm{CpG}(n=8)$, ablation $+\mathrm{CpG}+\alpha \mathrm{PD}-1(n=18), \mathrm{CpG}+\alpha \mathrm{PD}-1(n=21), \mathrm{CpG}+\alpha \mathrm{PD}-1-$ Prime $(n=21)$, ablation $+\mathrm{CpG}$ $+\alpha \mathrm{PD}$-1-Prime $(n=28)$, Multisite $\mathrm{CpG}+\alpha \mathrm{PD}$-1-Prime $(n=4)$, and Multisite ablation $+\mathrm{CpG}+\alpha \mathrm{PD}-1$ Prime $(n=5)$. All injections below immediately followed ablation. Tumor diameters were measured with ultrasound biweekly, and mice were sacrificed when tumors reached $1.5 \mathrm{~cm}$ in the longest dimension. For all relevant groups, $\alpha \mathrm{PD}-1$ was injected i.p. at a dose of $200 \mu \mathrm{g}$, as in ref. 41 . For all relevant groups, CpG was injected i.t. into a single tumor at a dose of $100 \mu \mathrm{g}$, as in ref. 7 .

For the ablation, ablation $+\mathrm{CpG}$ and ablation $+\mathrm{CpG}+\alpha \mathrm{PD}-1$ groups, MRgFUS ablation was performed in a single tumor on days 21 and 28 after initial tumor implantation. For CpG, ablation $+\mathrm{CpG}, \mathrm{CpG}$ $+\alpha \mathrm{PD}-1$, and ablation $+\mathrm{CpG}+\alpha \mathrm{PD}-1$ groups, $\mathrm{CpG}$ was injected into a single tumor on days 21, 24, and 28. For the $\alpha \mathrm{PD}-1, \mathrm{CpG}+\alpha \mathrm{PD}-1$, and ablation $+\mathrm{CpG}+\alpha \mathrm{PD}-1$ groups, $\alpha \mathrm{PD}-1$ was injected on days 21 and 28.

For 2 protocols that involved priming the immune system prior to ablation $(\mathrm{CpG}+\alpha \mathrm{PD}-1$-Prime and ablation $+\mathrm{CpG}+\alpha \mathrm{PD}-1-\mathrm{Prime}), \mathrm{CpG}$ was injected into a single tumor on days $21,24,28,31,38$, and 45 ; $\alpha \mathrm{PD}-1$ was injected on days 21,28 , and 35 ; and MRgFUS ablation was administered on days 31,38 , and 45. For the Multisite ablation $+\mathrm{CpG}+\alpha \mathrm{PD}-1$-Prime cohort, the tumor transplanted in the left side ninth mammary fat pad received CpG injections on days 21, 24, 28, 31, and 45 and ablation on days 31 and 45. The tumor transplanted in the fourth mammary fat pad received $1 \mathrm{CpG}$ injection and ablation treatment on day 38. $\alpha$ PD-1 was injected on days 21,28 , and 35 .

Flow cytometry antibodies. The following fluorochrome-conjugated mAbs were purchased from BioLegend: Pacific blue (PB) anti-CD45 (30-F11), fluorescein isothiocyanate (FITC) anti-F4/80 (BM8), phycoerythrin (PE) anti-NK1.1 (PK136), PE-Cy7-anti-CD3 (145-2C11), PE-Cy7 anti-CD11c (N418), allophycocyanin (APC) $\alpha$ PD-1 (RMP1-30), APC anti-CD206 (C068C2), APC-Cy7 anti-CD11b (M1/70), APC-Cy7 antiCD25 (PC61), Alexa Fluor (AF) 700-anti-CD8a (53-6.7), and AF-700 anti-Ly6G/Ly6C (Gr-1, RB6-8C5); the following were purchased from BD Biosciences: FITC anti-CD4 (GK1.5), and PE anti-CD86 (GL1); and the following were purchased from eBioscience: PE anti-Foxp3 (FJK-16s), PE-Cy5 anti-MHCII (M5/114.15.2), 
and PE anti-PD-L1 (MIH5). Isotype-matched mouse, rat, and hamster IgG mAbs were used as negative staining controls. In order to block FcyIII/II receptor-mediated unspecific binding, the anti-CD16/CD32 antibody (2.4G2) from BD Biosciences was used.

Flow cytometry. Bilateral tumor-bearing mice were sacrificed after the indicated treatment period for immune cell profiling via flow cytometry. Antibody combinations used to distinguish immune cell populations were $\mathrm{CD}_{4} 5^{+}$(leukocytes) plus the following: $\mathrm{CD}^{+}, \mathrm{CD}^{+}\left(\mathrm{CD} 4^{+} \mathrm{T}\right.$ cells), $\mathrm{CD}^{+}, \mathrm{CD}^{+}, \mathrm{PD}-1^{+}$ (PD-1 expressing CD4 ${ }^{+} \mathrm{T}$ cells), $\mathrm{CD}^{+}, \mathrm{CD}^{+}$(CD8 ${ }^{+} \mathrm{T}$ cells), $\mathrm{CD}^{+}, \mathrm{CD}^{+}, \mathrm{PD}-1^{+}$(PD-1 expressing CD8 ${ }^{+}$ $\mathrm{T}$ cells), $\mathrm{CD}^{+}, \mathrm{CD}^{2} 5^{+}, \mathrm{Foxp}^{+}$(Tregs), CD4 ${ }^{+}, \mathrm{CD}^{2} 5^{+}$, Foxp3 $3^{+}, \mathrm{PD}-1^{+}$(PD-1 expressing Tregs), CD11b ${ }^{+}$ $\mathrm{F} 4 / 80^{+}, \mathrm{Gr}-1^{-}$(macrophages), $\mathrm{CD} 11 \mathrm{~b}^{+}, \mathrm{F} 4 / 80^{+}, \mathrm{Gr}-1^{-}, \mathrm{CD}^{+} 6^{+}, \mathrm{MHCII}^{\mathrm{hi}}$ (M1 macrophages), $\mathrm{CD} 11 \mathrm{~b}^{+}$,

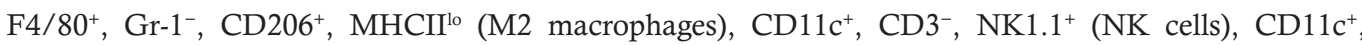
$\mathrm{MHCII}^{+}, \mathrm{F} 4 / 80^{-}$(DCs), CD11b ${ }^{+}, \mathrm{Gr}-1^{+}$(MDSCs), and PD-L1+ (PD-L1+ leukocytes).

Intracellular mouse Foxp3 staining was carried out using the eBioscience Anti-Mouse/Rat Foxp3 Staining Set (72-5775) following the manufacturer's instructions. All cell preparations were fixed in Cytofix buffer (BD Biosciences) diluted to 1\% paraformaldehyde (PFA) in PBS. Stained cells were analyzed within 24 hours on a LSRII flow cytometer (BD Biosciences), and all datasets were analyzed using FlowJo software vX (Tree Star Inc.).

IFN- $\gamma$ secretion from $\mathrm{CD}^{+}$and $\mathrm{CD}^{+} \mathrm{T}$ cells was quantified using the Mouse IFN- $\gamma$ Secretion Assay Cell Enrichment and Detection Kit (130-090-517, Miltenyi Biotec) according to the manufacturer's instructions.

PET imaging. Tumor permeability and the distribution of albumin was investigated using PET. Fatty acid and globumin-free BSA (A0281) was purchased from Sigma-Aldrich (99\%, MW of $67 \mathrm{kDa}$ ). In vivo and ex vivo imaging was performed with a micro-PET scanner (Focus 120, Siemens Medical Solutions Inc). Animals that received radiolabeled albumin were imaged at $0,6,18,24$, and 48 hours after tail-vein injection. Data were acquired for 2 hours and rebinned into a dynamic scan for the 0 - to 2-hour time point.

Cellular HMGB1 release. NDL tumor cells were plated at $3.5 \times 10^{5}$ cells/well in 12 -well tissue culture treated plates 24 hours prior to experiments. Cell plates were placed in either a $37^{\circ} \mathrm{C}, 60^{\circ} \mathrm{C}, 70^{\circ} \mathrm{C}, 80^{\circ} \mathrm{C}$, or $90^{\circ} \mathrm{C}$ water bath for 1 minute and allowed to cool to room temperature for 5 minutes. Doxorubicin $(5 \mu \mathrm{g} /$ $\mathrm{ml}$ final concentration) or media alone was then added directly to the cell wells to bring the total volume to $500 \mu$. Cells were incubated continuously with treatments for 24 hours in a $37^{\circ} \mathrm{C}$ humidified $\mathrm{CO}_{2}$ incubator. After 24 hours, cell culture media was collected and centrifuged at $300 \mathrm{~g}$ for 5 minutes. Debris-free culture supernatant was transferred to a fresh tube, and HMGB1 content in undiluted samples was measured via ELISA (MBS722248, MyBioSource) following the manufacturer's instructions. HMGB1 concentration was normalized to total cell number (live + dead cells, counted via hemocytometer) for each sample. All treatments were analyzed in triplicate.

Statistics. Statistical analyses were performed using Prism 7 software (GraphPad Software Inc.). Results are presented as mean \pm SEM, unless otherwise indicated. For analysis of 3 or more groups, a one-way ANOVA test was performed followed by a Fisher's LSD test without multiple comparisons correction in GraphPad Prism. Analysis of differences between 2 normally distributed test groups was performed using an unpaired $t$ test assuming unequal variance. $P$ values less than 0.05 were considered significant.

Study approval. All animal studies were conducted according to guidelines approved by the University of California, Davis Animal Care and Use Committee (IACUC).

\section{Author contributions}

MTS designed and conducted experiments, performed the analysis, and wrote the manuscript; ESI, AK, KDW, AWW, and STT conducted experiments and performed analysis; LMM and SMT maintained mouse models and provided assistance with design of experiments and MRgFUS ablations. YL and BZF conducted MRgFUS ablation. NEH, AMM, WJM, and ADB designed experiments. KWF planned and initiated the project, designed experiments, wrote the manuscript, and supervised the project.

\section{Acknowledgments}

We acknowledge the support of the Focused Ultrasound Surgery Foundation and NIHR01CA134659, NIHR01CA210553, and NIHT32GM099608. Imaging was performed the Center for Molecular and Genomic Imaging (CMGI), University of California, Davis. This project was supported by the University of California, Davis, Flow Cytometry Shared Resource Laboratory with funding from the NCI P30 
CA093373, and NIH NCRR C06-RR12088, S10 RR12964, and S10 RR 026825 grants and with technical assistance from Bridget McLaughlin and Jonathan Van Dyke.

Address correspondence to: Katherine W. Ferrara, Department of Biomedical Engineering, One Shields Avenue, University of California, Davis, Davis, California 95616, USA. Phone: 530.754.9436; E-mail: kwferrara@ucdavis.edu.

1. Topalian SL, Drake CG, Pardoll DM. Immune checkpoint blockade: a common denominator approach to cancer therapy. Cancer Cell. 2015;27(4):450-461.

2. Nguyen LT, Ohashi PS. Clinical blockade of PD1 and LAG3--potential mechanisms of action. Nat Rev Immunol. 2015;15(1):4556.

3. Postow MA, Callahan MK, Wolchok JD. Immune Checkpoint Blockade in Cancer Therapy. J Clin Oncol. 2015;33(17):19741982.

4. Nowak AK, Robinson BW, Lake RA. Synergy between chemotherapy and immunotherapy in the treatment of established murine solid tumors. Cancer Res. 2003;63(15):4490-4496.

5. Baxevanis CN, Perez SA, Papamichail M. Combinatorial treatments including vaccines, chemotherapy and monoclonal antibodies for cancer therapy. Cancer Immunol Immunother. 2009;58(3):317-324.

6. Steer HJ, Lake RA, Nowak AK, Robinson BW. Harnessing the immune response to treat cancer. Oncogene. 2010;29(48):63016313.

7. Marabelle A, et al. Depleting tumor-specific Tregs at a single site eradicates disseminated tumors. J Clin Invest. 2013;123(6):2447-2463.

8. Davila E, Kennedy R, Celis E. Generation of antitumor immunity by cytotoxic T lymphocyte epitope peptide vaccination, CpG-oligodeoxynucleotide adjuvant, and CTLA-4 blockade. Cancer Res. 2003;63(12):3281-3288.

9. Formenti SC, Demaria S. Combining radiotherapy and cancer immunotherapy: a paradigm shift. J Natl Cancer Inst. 2013;105(4):256-265.

10. Vanpouille-Box C, Pilones KA, Wennerberg E, Formenti SC, Demaria S. In situ vaccination by radiotherapy to improve responses to anti-CTLA-4 treatment. Vaccine. 2015;33(51):7415-7422.

11. Nesslinger NJ, et al. Standard treatments induce antigen-specific immune responses in prostate cancer. Clin Cancer Res. 2007;13(5):1493-1502.

12. Schaue D, et al. T-cell responses to survivin in cancer patients undergoing radiation therapy. Clin Cancer Res. 2008;14(15):4883-4890.

13. Burnette B, Fu YX, Weichselbaum RR. The confluence of radiotherapy and immunotherapy. Front Oncol. 2012;2:143.

14. Smyth MJ, Ngiow SF, Ribas A, Teng MW. Combination cancer immunotherapies tailored to the tumour microenvironment. Nat Rev Clin Oncol. 2016;13(3):143-158.

15. Wu F. High intensity focused ultrasound ablation and antitumor immune response. J Acoust Soc Am. 2013;134(2):1695-1701

16. Unga J, Hashida M. Ultrasound induced cancer immunotherapy. Adv Drug Deliv Rev. 2014;72:144-153.

17. Kennedy JE. High-intensity focused ultrasound in the treatment of solid tumours. Nat Rev Cancer. 2005;5(4):321-327.

18. Peek MC, et al. Systematic review of high-intensity focused ultrasound ablation in the treatment of breast cancer. Br J Surg. 2015;102(8):873-82; discussion 882.

19. Zhou YF. High intensity focused ultrasound in clinical tumor ablation. World J Clin Oncol. 2011;2(1):8-27.

20. Tempany CM, Stewart EA, McDannold N, Quade BJ, Jolesz FA, Hynynen K. MR imaging-guided focused ultrasound surgery of uterine leiomyomas: a feasibility study. Radiology. 2003;226(3):897-905.

21. Ikink ME, et al. Volumetric MR-guided high-intensity focused ultrasound versus uterine artery embolisation for treatment of symptomatic uterine fibroids: comparison of symptom improvement and reintervention rates. Eur Radiol. 2014;24(10):2649-2657

22. Galiana G, Branca RT, Jenista ER, Warren WS. Accurate temperature imaging based on intermolecular coherences in magnetic resonance. Science. 2008;322(5900):421-424.

23. Quesson B, de Zwart JA, Moonen CT. Magnetic resonance temperature imaging for guidance of thermotherapy. J Magn Reson Imaging. 2000;12(4):525-533.

24. Dewhirst MW, Vujaskovic Z, Jones E, Thrall D. Re-setting the biologic rationale for thermal therapy. Int J Hyperthermia. 2005;21(8):779-790

25. Wong AW, et al. Ultrasound ablation enhances drug accumulation and survival in mammary carcinoma models. J Clin Invest. 2016;126(1):99-111.

26. Cardiff RD, et al. Quantitation of fixative-induced morphologic and antigenic variation in mouse and human breast cancers. Lab Invest. 2013;93(4):480-497.

27. Miller JK, et al. Suppression of the negative regulator LRIG1 contributes to ErbB2 overexpression in breast cancer. Cancer Res. 2008;68(20):8286-8294.

28. Hogan BV, Peter MB, Shenoy HG, Horgan K, Hughes TA. Surgery induced immunosuppression. Surgeon. 2011;9(1):38-43.

29. Gulley JL. Developing immunotherapy strategies in the treatment of prostate cancer. Asian Journal of Urology. 2016;3(4): 278285.

30. Grosso JF, Jure-Kunkel MN. CTLA-4 blockade in tumor models: an overview of preclinical and translational research. Cancer Immun. 2013;13:5.

31. Monjazeb AM, et al. Blocking Indolamine-2,3-Dioxygenase Rebound Immune Suppression Boosts Antitumor Effects of Radio-Immunotherapy in Murine Models and Spontaneous Canine Malignancies. Clin Cancer Res. 2016;22(17):4328-4340. 
32. Dewan $\mathrm{MZ}$, et al. Fractionated but not single-dose radiotherapy induces an immune-mediated abscopal effect when combined with anti-CTLA-4 antibody. Clin Cancer Res. 2009;15(17):5379-5388.

33. Dovedi SJ, et al. Systemic delivery of a TLR7 agonist in combination with radiation primes durable antitumor immune responses in mouse models of lymphoma. Blood. 2013;121(2):251-259.

34. den Brok MH, et al. In situ tumor ablation creates an antigen source for the generation of antitumor immunity. Cancer Res. 2004;64(11):4024-4029.

35. Hata AN, et al. Tumor cells can follow distinct evolutionary paths to become resistant to epidermal growth factor receptor inhibition. Nat Med. 2016;22(3):262-269.

36. McGranahan N, Favero F, de Bruin EC, Birkbak NJ, Szallasi Z, Swanton C. Clonal status of actionable driver events and the timing of mutational processes in cancer evolution. Sci Transl Med. 2015;7(283):283ra54

37. Helissey C, Vicier C, Champiat S. The development of immunotherapy in older adults: New treatments, new toxicities? J Geriatr Oncol. 2016;7(5):325-333.

38. Siegel PM, Ryan ED, Cardiff RD, Muller WJ. Elevated expression of activated forms of Neu/ErbB-2 and ErbB-3 are involved in the induction of mammary tumors in transgenic mice: implications for human breast cancer. EMBO J. 1999;18(8):2149-2164

39. Borowsky AD, et al. Syngeneic mouse mammary carcinoma cell lines: two closely related cell lines with divergent metastatic behavior. Clin Exp Metastasis. 2005;22(1):47-59.

40. Fite BZ, et al. Magnetic resonance thermometry at 7T for real-time monitoring and correction of ultrasound induced mild hyperthermia. PLoS One. 2012;7(4):e35509.

41. Gubin MM, et al. Checkpoint blockade cancer immunotherapy targets tumour-specific mutant antigens. Nature 2014;515(7528):577-581. 